\title{
EXPONENTIAL STABILITY OF NONMONOTONE TRAVELING WAVES FOR NICHOLSON'S BLOWFLIES EQUATION*
}

\author{
CHI-KUN LIN ${ }^{\dagger}$, CHI-TIEN LIN, YANPING LIN§, AND MING MEI \\ Dedicated to Professor I-Liang Chern on his 60th birthday
}

\begin{abstract}
This paper is concerned with Nicholson's blowflies equation, a kind of time-delayed reaction-diffusion equation. It is known that when the ratio of birth rate coefficient and death rate coefficient satisfies $1<\frac{p}{d} \leq e$, the equation is monotone and possesses monotone traveling wavefronts, which have been intensively studied in previous research. However, when $\frac{p}{d}>e$, the equation losses its monotonicity, and its traveling waves are oscillatory when the time-delay $r$ or the wave speed $c$ is large, which causes the study of stability of these nonmonotone traveling waves to be challenging. In this paper, we use the technical weighted energy method to prove that when $e<\frac{p}{d} \leq e^{2}$, all noncritical traveling waves $\phi(x+c t)$ with $c>c_{*}>0$ are exponentially stable, where $c_{*}>0$ is the minimum wave speed. Here, we allow the traveling wave to be either monotone or nonmonotone with any speed $c>c_{*}$ and any size of the time-delay $r>0$; however, when $\frac{p}{d}>e^{2}$ with a small time-delay $r<\left[\pi-\arctan \sqrt{\ln \frac{p}{d}\left(\ln \frac{p}{d}-2\right)}\right] / d \sqrt{\ln \frac{p}{d}\left(\ln \frac{p}{d}-2\right)}$, all noncritical traveling waves $\phi(x+c t)$ with $c>c_{*}>0$ are exponentially stable, too. As a corollary, we also prove the uniqueness of traveling waves in the case of $\frac{p}{d}>e^{2}$, which to the best of our knowledge was open. Finally, some numerical simulations are carried out. When $e<\frac{p}{d} \leq e^{2}$, we demonstrate numerically that after a long time the solution behaves like a monotone traveling wave for a small time-delay, and behaves like an oscillatory traveling wave for a big time-delay. When $\frac{p}{d}>e^{2}$, if the time-delay is small, then the solution numerically behaves like a monotone/nonmonotone traveling wave, but if the time-delay is big, then the solution is numerically demonstrated to be chaotically oscillatory but not an oscillatory traveling wave. These either confirm and support our theoretical results or open up some new phenomena for future research.
\end{abstract}

Key words. Nicholson's blowflies equation, time-delayed reaction-diffusion equation, nonmonotone traveling waves, stability

AMS subject classifications. 35K57, 34K20, 92D25

DOI. $10.1137 / 120904391$

* Received by the editors January 2, 2013; accepted for publication (in revised form) December 12, 2013; published electronically March 6, 2014.

http://www.siam.org/journals/sima/46-2/90439.html

$\dagger$ Department of Applied Mathematics, National Chiao Tung University, Hsinchu 30010, Taiwan, R.O.C. (cklin@math.nctu.edu.tw). This author's research was supported in part by the National Science Council of Taiwan, R.O.C., under grant 101-2115-M-009-008-MY2.

$\ddagger$ Department of Financial And Computational Mathematics, Providence University, Taichung 43301, Taiwan, R.O.C. (ctlin@pu.edu.tw). This author's research was supported in part by the National Science Council of Taiwan, R.O.C., under grant NSC99-2115-M-126-002-MY2 and NSC1012115-M-126-001.

$\S$ Department of Applied Mathematics, The Hong Kong Polytechnic University, Hung Hum, Hong Kong, China, and Department of Mathematical and Statistical Sciences, University of Alberta, Edmonton, Alberta T6G 2G1, Canada (malin@polyu.edu.hk, yanlin@ualberta.ca). This author's research was supported in part by GRF grant of Hong Kong B-Q30J, Hong Kong Polyu G-YJ92, and the NSERC of Canada.

『Corresponding author. Department of Mathematics, Champlain College Saint-Lambert, SaintLambert, Quebec J4P 3P2, Canada, and Department of Mathematics and Statistics, McGill University, Montreal, Quebec H3A 2K6, Canada (ming.mei@mcgill.ca, mmei@champlaincollege.qc.ca). This author's research was supported in part by NSERC grant RGPIN 354724-11, FQRNT grant 164832, and the project "Collaboration internationale de la recherche scientifique à Hong Kong et Japon" from Cégep International. 
1. Introduction and main results. We consider a time-delayed reactiondiffusion equation

$$
\frac{\partial v(t, x)}{\partial t}-D \frac{\partial^{2} v(t, x)}{\partial x^{2}}+d v(t, x)=b(v(t-r, x)), \quad(t, x) \in R_{+} \times R
$$

with the initial data

$$
v(s, x)=v_{0}(s, x), \quad s \in[-r, 0], x \in R .
$$

This model represents the population distribution of single species such as the Australian blowfly $[10,11,16,28,34]$, which is derived from the original delay ODE model [12] based on Nicholson's experimental data [30,31]. Here, $v(t, x)$ denotes the mature population at time $t$ and location $x ; D>0$ is the spatial diffusion rate of the mature population; $d>0$ is the death rate of the mature population; $r>0$ is the maturation delay, the time required for a newborn to become matured; and $b(v)$ is the birth rate function satisfying

$\left(\mathrm{H}_{1}\right)$ two constant equilibria of (1.1): $v_{-}=0$ is unstable and $v_{+}$is stable, namely, $b(0)=0, d v_{+}=b\left(v_{+}\right), d-b^{\prime}(0)<0$, and $d-b^{\prime}\left(v_{+}\right)>0$

$\left(\mathrm{H}_{2}\right)$ the unimodality condition: $b(v)>0$ has only one positive local maximum at the point $v_{*} \in\left(0, v_{+}\right)$, and $b(v)$ is increasing on $\left[0, v_{*}\right]$ and decreasing on $\left[v_{*},+\infty\right)$, which also implies $b^{\prime}(0)>0$ and $b^{\prime}\left(v_{+}\right)<0$;

$\left(\mathrm{H}_{3}\right) \quad b \in C^{2}[0, \infty)$ and $\left|b^{\prime}(v)\right| \leq b^{\prime}(0)$ for $v \in[0, \infty)$.

A typical example is the so-called Nicholson's birth rate function

$$
b(v)=p v e^{-a v}, \quad a>0, p>0,
$$

where $p>0$ is the impact of the birth on the immature population. Equation (1.1) with the particular birth rate function (1.3) is called Nicholson's blowflies equation.

Clearly, the so-called Nicholson's blowflies equation possesses two constant equilibria,

$$
v_{-}=0 \text { and } v_{+}=\frac{1}{a} \ln \frac{p}{d},
$$

and $v_{+}>0$ for $\frac{p}{d}>1$. Linearizing (1.1) without the delay $(r=0)$ around $v_{ \pm}$, and setting $\bar{v}=v-v_{ \pm}$, we get

$$
\bar{v}_{t}-D \bar{v}_{x x}=\left(b^{\prime}\left(v_{ \pm}\right)-d\right) \bar{v} .
$$

Notice that $b^{\prime}\left(v_{-}\right)-d=p-d>0$ and $b^{\prime}\left(v_{+}\right)-d=-d \ln \frac{p}{d}<0$, so $v_{-}=0$ is an unstable node of (1.4), and $v_{+}=\frac{1}{a} \ln \frac{p}{d}$ is a stable node of (1.4). Thus, Nicholson's blowflies equation (1.1) possesses one unstable node $v_{-}$and one stable node $v_{+}$. Such an equation is called a monostable equation, like the classic Fisher-KPP equation

$$
v_{t}-v_{x x}=v(1-v) .
$$

The traveling wavefronts for (1.1) connecting with $v_{ \pm}$at far fields are the special solutions to (1.1) in the form of $\phi(x+c t) \geq 0$, namely,

$$
\left\{\begin{array}{l}
c \phi^{\prime}(\xi)-D \phi^{\prime \prime}(\xi)+d \phi(\xi)=b(\phi(\xi-c r)), \\
\phi( \pm \infty)=v_{ \pm}
\end{array}\right.
$$

where $\xi=x+c t,{ }^{\prime}=\frac{d}{d \xi}$, and $c$ is the wave speed. 
The main purpose of this paper is to investigate the stability of the traveling waves, in particular for the challenging case of oscillatory traveling waves when the equation is nonmonotone.

First of all, let us provide some background on the existence of traveling waves $\phi(x+c t)$ of $(1.5)$. When $1<\frac{p}{d} \leq e$, the birth rate function $b(v)=p v e^{-a v}$ is monotonically increasing for $v \in\left[0, v_{+}\right]$. As shown in [35], So and Zou proved by the upper-lower solutions method that there exists a minimal wave speed $c_{*}=c_{*}(r)>0$ (the so-called critical wave speed, which is given by the characteristic equation of the linearizing equation of (1.5) around the equilibrium $v_{-}=0$ ), when $c \geq c_{*}$, for any time-delay $r>0$, the traveling wavefronts $\phi(x+c t)$ exist and are monotone. The uniqueness (up to a constant shift) was shown by Aguerrea, Gomez, and Trofimchuk [1] recently by means of the Diekmann-Kaper theory. However, when $\frac{p}{d}>e$, the birth rate $b(v)$ is nonmonotone on $\left[0, v_{+}\right]$, where $b(v)$ is increasing in $\left(0, \frac{1}{a}\right)$ and decreasing in $\left(\frac{1}{a}, v_{+}\right)$; then the story may be totally different and quite challenging, because the waves may not be monotone but cross-oscillatory as numerically indicated. Unfortunately, the simple but useful method of upper-lower-solutions has failed to prove the existence of traveling waves. In this case, Faria, Huang, and Wu [4] applied the Lyapunov-Schmidt reduction method together with the perturbation argument to show the existence of traveling waves under certain conditions, that is, when the time-delay $r$ is small, i.e., $r \ll 1$; then the traveling waves $\phi(x+c t)$ of $(1.5)$ with a large speed $c \gg c_{*}>0$ exist. This work was then immediately improved upon by Faria and Trofimchuk [5,6] through a detailed analysis of heteroclinic solutions, and by Ma [19], who constructed two auxiliary functions and applied Schauder's fixedpoint theorem, namely, for $e<\frac{p}{d} \leq e^{2}$ with any size of time-delay $r$, the traveling waves $\phi(x+c t)$ with $c \geq c_{*}>0$ exist. The waves are nonmonotone either for a large time-delay $r$ such that

$$
d\left(\ln \frac{p}{d}-1\right) r e^{d r+1}>1
$$

or for a large speed $c>c^{*}$, where $c^{*}=c^{*}(r)>0$ is given by the characteristic equation of the linearizing equation of (1.5) around the equilibrium $v_{+}$. Remarkably, Gomez and Trofimchuk [7] recently showed that, for $e<\frac{p}{d} \leq e^{2}$, the waves are monotone if and only if $(c, r)$ is in a certain region $\mathcal{D}$ (see Figure 1), i.e., $c \in\left[c_{*}(r), c^{*}(r)\right]$ with some $r>0$. Precisely, let $\underline{r}>0$ be the root of the equation

$$
d\left(\ln \frac{p}{d}-1\right) \underline{r} e^{d \underline{r}+1}=1,
$$

which is also the critical point for the solution possibly to cause oscillations for the linear delay ODE [36]

$$
v^{\prime}(t)+d v(t)=b^{\prime}\left(v_{+}\right) v(t-r)
$$

if $0<r<\underline{r}$, then $c^{*}=\infty$, and thus all traveling waves $\phi(x+c t)$ are monotone for $c \geq c_{*}$. Furthermore, when $r \geq \underline{r}$, if $\frac{p}{d}$ is close to $e$ such that $e=2.7819 \cdots<$ $\frac{p}{d} \leq \nu_{0}=2.808 \cdots$, then the wavefronts are still monotone for $c \in\left[c_{*}, c^{*}\right]$, where $\nu_{0}=2.808 \cdots$ is the largest number for $\frac{p}{d}$ such that $c_{*}(r)=c^{*}(r)$ has no solution. However, if $\nu_{0}<\frac{p}{d} \leq e^{2}$, then there exists $r_{0}>0$, which is the unique intersection point of $c_{*}(r)$ and $c^{*}(r)$ and satisfies $r_{0}>\underline{r}$, such that the wavefronts are monotone for $(c, r) \in\left[c_{*}, c^{*}\right] \times\left[\underline{r}, r_{0}\right]$. In the other case, when $(c, r)$ is out of the mentioned region $\mathcal{D}$, the traveling waves are slowly oscillating around $v_{+}$. The uniqueness of the 

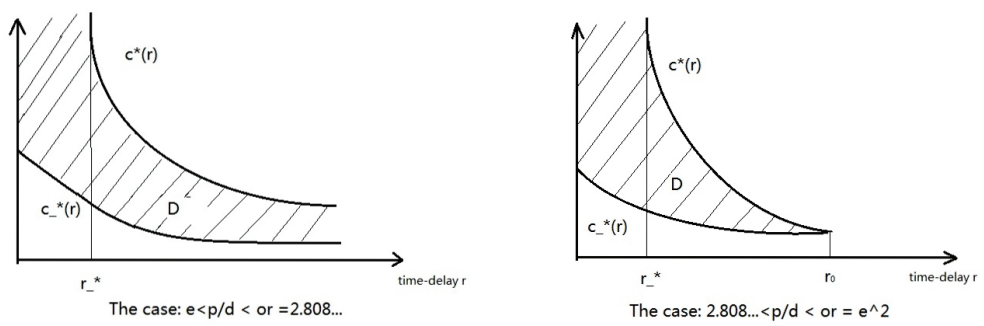

FIG. 1. Domain of $(c, r) \in D$ for monotone traveling waves in the case of $e<\frac{p}{d} \leq e^{2}$.

waves up to shift in this case has also been proved by the Diekmann-Kaper theory in [1]. Finally, when $\frac{p}{d}>e^{2}$, the wavefronts exist only for $r$ in some bounded set [38, 37], and the wavefronts are slowly oscillating at $+\infty$ when the time-delay $r$ satisfies (1.6). Notice that, as specified in [37], if $r \gg 1$, even though $c>c_{*}$, there will be no traveling waves, or if $c \gg 1$, there also will be no traveling waves. However, the study in this case is quite incomplete and leaves many questions unanswered. For example, can uniqueness be proved for this case? what is the necessary condition for the nonexistence of traveling waves? and so on. Here, as a corollary of our stability theorem, we will give a positive answer concerning the uniqueness of traveling waves. follows.

Now we summarize the existence and uniqueness of traveling waves of (1.5) as

ThEOREM 1.1 (existence of traveling waves). Let $b(v)=p v e^{-a v}$.

1. When $1<\frac{p}{d} \leq e$, then there exist two numbers $c_{*}>0$ and $\lambda_{*}=\lambda_{*}\left(c_{*}\right)>0$, which are given implicitly by

$$
c_{*} \lambda_{*}-D \lambda_{*}^{2}+d=b^{\prime}(0) e^{-\lambda_{*} c_{*} r} \text { and } c_{*}-2 D \lambda_{*}=-b^{\prime}(0) c_{*} r e^{-\lambda_{*} c_{*} r},
$$

with $b^{\prime}(0)=p$, such that

(a) for $c<c_{*}$, no traveling waves $\phi(x+c t)$ exist;

(b) for $c \geq c_{*}$, the traveling waves $\phi(x+c t)$ of (1.5) exist and are unique up to shift. Particularly, when $c>c_{*}$, there exist two numbers depending on the speed $c, \lambda_{1}=\lambda_{1}(c)>0$ and $\lambda_{2}=\lambda_{2}(c)>0$, as the positive roots of the equation

$$
c \lambda_{i}-D \lambda_{i}^{2}+d=b^{\prime}(0) e^{-\lambda_{i} c r}, i=1,2,
$$

satisfying

$$
c \lambda-D \lambda^{2}+d>b^{\prime}(0) e^{-\lambda c r} \quad \text { for } \lambda_{1}<\lambda<\lambda_{2} .
$$

2. When $e<\frac{p}{d} \leq e^{2}$, the traveling waves exist and are unique up to shift for any given time-delay $r>0$. Namely, there exist two numbers $c_{*}>0$ and $\lambda_{*}$, which are the same as in (1.9), and the inequality (1.11) still holds, such that

(a) for $c<c_{*}$, no traveling waves $\phi(x+c t)$ exist;

(b) for $c \geq c_{*}$, the traveling waves $\phi(x+c t)$ of (1.5) exist and are unique up to shift. Particularly, $\phi(x+c t)$ is monotone for $(c, r)$ in a certain region $\mathcal{D}$ and nonmonotone for $(c, r)$ out of $\mathcal{D}$ (see Figure 1). Precisely,

(i) if $0<r<\underline{r}$, then $\phi(x+c t)$ is always monotone for $c \geq c_{*}$; 
(ii) if e $<\frac{p}{d} \leq \nu_{0}=2.808 \cdots$ even if $r \geq \underline{r}$ with $c \in\left[c_{*}, c^{*}\right]$, then $\phi(x+c t)$ is monotone, where $c_{*}=c_{*}(r)$ is given by (1.9), and $c^{*}=c^{*}(r)$ is determined by

$$
c^{*} \lambda^{*}-D \lambda^{* 2}+d=b^{\prime}\left(v_{+}\right) e^{-\lambda^{*} c^{*} r}, c^{*}-2 D \lambda^{*}=-b^{\prime}\left(v_{+}\right) c^{*} r e^{-\lambda^{*} c^{*} r}
$$$$
\text { with } b^{\prime}\left(v_{+}\right)=d\left(1-\ln \frac{p}{d}\right) \text {; }
$$

(iii) if $\nu_{0}<\frac{p}{d} \leq e^{2}$ and $r \in\left[\underline{r}, r_{0}\right]$ with $c \in\left[c_{*}, c^{*}\right]$, then $\phi(x+c t)$ is monotone, where $r_{0}>\underline{r}$ is the unique intersection point of $c_{*}(r)$ and $c^{*}(r)$;

(iv) otherwise, when $(c, r) \notin \mathcal{D}$, then $\phi(x+c t)$ is nonmonotone, and slowly oscillates around $v_{+}$.

3. When $\frac{p}{d}>e^{2}$ and $0<r \ll 1$, then the traveling waves $\phi(x+c t)$ exist for $c_{*} \leq c \leq c^{*}$ and are slowly oscillating around $v_{+}$. If $r \gg 1$ or $c \gg 1$, no traveling waves exist. No uniqueness of the wavefronts in this case is proved at this moment.

As mentioned before, the main goal of this paper is to show that these traveling waves, including nonmonotone waves, are asymptotically stable as $t \rightarrow \infty$. Here, let us provide further details on the progress of stability of the traveling waves in this direction. When $1<\frac{p}{d} \leq e$, for the monotone traveling waves $\phi(x+c t)$ of $(1.5)$, Schaaf [32] in 1987 first studied the linearized stability of wavefronts for time-delayed monostable equations using a spectral method. Since then, this topic was barely touched upon until 2004, when Mei et al. [28] showed nonlinear (local) stability of the wavefronts by a technical weighted energy method. Then Mei and coauthors $[17,23,24,27]$ further obtained global stability using both the weighted energy method and the comparison principle. These results were then extended to more general timedelayed reaction-diffusion equations with monostability by $\mathrm{Lv}$ and Wang [18] and $\mathrm{Wu}$, $\mathrm{Li}$, and Liu [39]. Recently, the global stability of critical wavefronts $\phi\left(x+c_{*} t\right)$ with optimal convergent rates was obtained by Mei, Ou, and Zhao [25] and Mei and Wang [29] using the Fourier transform and Green's function method plus energy estimates. Notice that these stabilities rely on the monotonicity of both the equation and the wavefronts. However, when $\frac{p}{d}>e$, equation (1.1) lacks monotonicity and the traveling waves may oscillate around $v_{+}$; research on the stability of such oscillatory waves was only recently carried out in [40]. Because of the lack of monotonicity, the equation doesn't possess the comparison principle, and we cannot expect global stability. But we may still be able to get local stability, because the weighted energy method doesn't require the monotonicity of the equations and works for any nonmonotone equation if it possesses some viscosity or a damping or relaxation effect [3, 14, 20, 26]. Under such considerations, recently $\mathrm{Wu}$, Zhao, and Liu [40] first showed asymptotic stability for nonmonotone traveling waves, but they needed to restrict $\frac{p}{d}$ to be sufficiently close to $e, \frac{p}{d} \approx e$, and the wave speed to be sufficiently large, $c \gg c_{*}$. It is interesting to note that the $L^{2}$-weighted energy method developed in our previous works [23, 28] is useful only when $e<\frac{p}{d}<e^{2}$, because a restriction $d-\left|b^{\prime}\left(v_{+}\right)\right|>0$ is necessary when we establish the desired $L^{2}$ energy estimate of the solution for $\phi(x+c t)$ near the farfield state $v_{+}$. Thus, such a restriction causes the stability of the waves in the case of $\frac{p}{d}>e^{2}$ to fail, and so the problem remains open. Another interesting and important issue is the stability of those slower waves with a small speed arbitrarily close to the minimum speed $c_{*}$, i.e., $c>c_{*}$ but $\left|c-c_{*}\right| \ll 1$, in particular the stability of critical wave $\phi\left(x+c_{*} t\right)$. Answering these questions will be the main task of the present paper. In fact, in this paper we will prove that for $e<\frac{p}{d} \leq e^{2}$ all traveling waves $\phi(x+c t)$ 
with $c>c_{*}$, including those oscillating waves, are exponentially stable when the initial perturbations around the waves in a certain weighted Sobolev space are small enough. Here, the time-delay $r>0$ can be allowed to be arbitrary. However, for $\frac{p}{d}>e^{2}$, if the time-delay $r$ satisfies $0<r<\left[\pi-\arctan \sqrt{\ln \frac{p}{d}\left(\ln \frac{p}{d}-2\right)}\right] / d \sqrt{\ln \frac{p}{d}\left(\ln \frac{p}{d}-2\right)}$, then all noncritical traveling waves, including the oscillating waves, are asymptotically stable, too. This condition on $r$ should be optimal, because it is the sufficient and necessary condition for stability of the solution to the linear delay differential equation (1.8); cf. [2]. The adopted approach for proving the stability of traveling waves in this paper is still the technical weighted energy method, but in order to treat the case when $\phi\left(x+c t\right.$ ) is near $v_{+}$(or, say, $x \rightarrow \infty$ ), in contrast to previous studies, we use the nonlinear Halanay's inequality (see Lemma 3.9 below) to get the desired exponential decay estimate. However, for $\frac{p}{d}>e^{2}$ with large time-delay $r \gg 1$, as we numerically demonstrate in the last section of the paper, the solution is chaotically oscillatory and doesn't behave like an oscillatory traveling wave (in fact, to the best of our knowledge, no traveling waves exist in this case). Such phenomena leave us with many challenges. Stability of the critical wave $\phi\left(x+c_{*} t\right)$ in the case of the nonmonotone equation unfortunately remains an open problem, since current methods have failed. So a new strategy has to be developed in the future.

Before stating our main result, let us make the following notation. Throughout the paper, $C>0$ always denotes a generic constant, while $C_{i}>0(i=0,1,2, \ldots)$ represents a specific constant. Let $I$ be an interval, typically $I=R . L^{2}(I)$ is the space of the square integrable functions defined on $I$, and $H^{k}(I)(k \geq 0)$ is the Sobolev space of the $L^{2}$-functions $f(x)$ defined on the interval $I$ whose derivatives $\frac{d^{i}}{d x^{2}} f(i=1, \ldots, k)$ also belong to $L^{2}(I)$. $L_{w}^{2}(I)$ denotes the weighted $L^{2}$-space with a weight function $w(x)>0$, and its norm is defined by

$$
\|f\|_{L_{w}^{2}}=\left(\int_{I} w(x)|f(x)|^{2} d x\right)^{1 / 2} .
$$

$H_{w}^{k}(I)$ is the weighted Sobolev space with the norm given by

$$
\|f\|_{H_{w}^{k}}=\left(\sum_{i=0}^{k} \int_{I} w(x)\left|\frac{d^{i}}{d x^{i}} f(x)\right|^{2} d x\right)^{1 / 2} .
$$

Let $T>0$ be a number and $\mathcal{B}$ a Banach space. We denote by $C([0, T] ; \mathcal{B})$ the space of the $\mathcal{B}$-valued continuous functions on $[0, T]$ and by $L^{2}([0, T] ; \mathcal{B})$ the space of the $\mathcal{B}$-valued $L^{2}$-functions on $[0, T]$.

Throughout this paper, we consider (1.1) with a bit general birth rate $b(v)$ satisfying $\left(\mathrm{H}_{1}\right)-\left(\mathrm{H}_{3}\right)$. Let $\phi(x+c t)$ be a given noncritical traveling wave with $c>c_{*}$, even if it is monotone or slowly oscillatory around $v_{+}$. Here, $c_{*}=c_{*}(r)$ and $\lambda_{*}=\lambda_{*}(r)$ satisfy (1.9) with a general unimodality $b(v)$, and, correspondingly, $c$ and $\lambda$ satisfy (1.11). We define a weight function related to such a number $\lambda>0$,

$$
w(x)=e^{-2 \lambda\left(x-x_{0}\right)}, \quad \lambda \in\left(\lambda_{1}, \lambda_{2}\right), \quad \text { with a sufficient large number } x_{0} \gg 1 .
$$

Now we state the stability of traveling waves for (1.1) with a general nonmonotone birth rate.

THEOREM 1.2 (stability of monotone/nonmonotone traveling waves). Let the birth rate function $b(v)$ be general and satisfy $\left(H_{1}\right)-\left(H_{3}\right)$, and assume either $d \geq$ 
$\left|b^{\prime}\left(v_{+}\right)\right|$with any time-delay $r>0$, or $d<\left|b^{\prime}\left(v_{+}\right)\right|$but with a small time-delay $0<$ $r<\bar{r}$, where

$$
\bar{r}:=\frac{\pi-\arctan \left(\sqrt{\left|b^{\prime}\left(v_{+}\right)\right|^{2}-d^{2}} / d\right)}{\sqrt{\left|b^{\prime}\left(v_{+}\right)\right|^{2}-d^{2}}} .
$$

For any given traveling wave $\phi(x+c t)$ with $c>c_{*}$ to (1.1), whether it is monotone or nonmonotone, suppose that $v_{0}(s, x)-\phi(x+c s) \in C\left([-r, 0] ; C(R) \cap H_{w}^{2}(R)\right) \cap$ $L^{2}\left([-r, 0] ; H_{w}^{2}(R)\right)$, and $\lim _{x \rightarrow+\infty}\left[v_{0}(s, x)-\phi(x+c s)\right]=: u_{0, \infty}(s) \in C[-r, 0]$ exists uniformly with respect to $s \in[-r, 0]$. There exist some constants $\delta_{0}>0,0<\mu_{2}=$ $\mu_{2}\left(p, d, r, b^{\prime}\left(v_{+}\right)\right)<d$, and $0<\mu=\mu\left(p, d, r, c, \lambda, b^{\prime}\left(v_{+}\right)\right)<\mu_{2}$, all independent of $x$, $t$, and $u(t, x)$, when the initial perturbation is small:

$$
\max _{s \in[-r, 0]}\left\|\left(v_{0}-\phi\right)(s)\right\|_{C}^{2}+\left\|\left(v_{0}-\phi\right)(0)\right\|_{H_{w}^{2}}^{2}+\int_{-r}^{0}\left\|\left(v_{0}-\phi\right)(s)\right\|_{H_{w}^{2}}^{2} d s \leq \delta_{0}^{2} .
$$

Then the solution $v(t, x)$ of (1.1) and (1.2) is unique, exists globally in time, and satisfies

$(1.15)$

$v(t, x)-\phi(x+c t) \in C\left([-r, \infty) ; C(R) \cap H_{w}^{2}(R)\right) \cap L^{2}\left([-r, \infty) ; H_{w}^{2}(R)\right) \cap \mathcal{C}_{u n i f}[-r, \infty)$

and

$$
\sup _{x \in R}|v(t, x)-\phi(x+c t)| \leq C e^{-\mu t}, \quad t>0,
$$

where $\mathcal{C}_{\text {unif }}[-r, T]$, for $0<T \leq \infty$, is defined by

$$
\begin{aligned}
\mathcal{C}_{\text {unif }}[-r, T]:= & \{u(t, x) \in C([-r, T] \times R) \text { such that } \\
& \lim _{x \rightarrow+\infty} e^{\mu_{2} t} u(t, x) \text { exists uniformly in } t \in[-r, T], \text { and } \\
& \lim _{x \rightarrow+\infty} u_{x}(t, x)=\lim _{x \rightarrow+\infty} u_{x x}(t, x)=0 \\
& \text { uniformly with respect to } t \in[-r, T]\} .
\end{aligned}
$$

Remark 1.

1. Notice that $w(x) \geq 1$ only for $x \leq x_{0}$, and $\lim _{x \rightarrow-\infty} w(x)=+\infty, \lim _{x \rightarrow \infty} w(x)$ $=0$. Thus, if $f \in H_{w}^{2}(R)$, then we cannot always guarantee the boundedness of $f$ as $x>x_{0}$. So, different from our previous works [23, 24, 27, 28] where the weight functions are selected to be greater than 1 for all $x$, and the initial perturbation in $H_{w}^{2}$ with $w \geq 1$ implies $\lim _{x \rightarrow \pm \infty}\left[v_{0}(s, x)-\phi(x+c s)\right]=0$, here we restrict the initial perturbation only in $C(R) \cap H_{w}^{1}$ for $w(+\infty)=0$, with a uniform convergence as $x \rightarrow+\infty$. This allows the initial perturbation to be uniformly bounded only at $x=\infty$ but may not be vanishing, namely, $\lim _{x \rightarrow \infty}\left[v_{0}(s, x)-\phi(x+c s)\right]=: u_{0, \infty}(s) \neq 0$.

2. Technically, in the later proof, we need to select the weight function $w(x)=$ $e^{-2 \lambda\left(x-x_{0}\right)}$ for $\lambda \in\left(\lambda_{1}, \lambda_{2}\right)$ (see (1.10)). This makes the initial perturbation around the wavefront like $\left|v_{0}(s, x)-\phi(x+c s)\right|=O(1) e^{-\lambda|x|}$ as $x \rightarrow-\infty$. If the initial perturbation decays much faster, namely, $\lambda \geq \lambda_{2}$, the initial perturbation $\left|v_{0}(s, x)-\phi(x+c s)\right|$ obviously belongs to $C\left([-r, 0] ; C(R) \cap H_{w}^{1}(R)\right)$; then the stability theorem, Theorem 1.2, directly implies the exponential convergence of the solution $v(t, x)$ to the traveling wave $\phi(x+c t)$. 
COROLlaRY 1.3 (uniqueness of traveling waves). Let the birth rate function $b(v)$ be general and satisfy $\left(H_{1}\right)-\left(H_{3}\right)$, and let either $d \geq\left|b^{\prime}\left(v_{+}\right)\right|$with any time-delay $r>0$, or $d<\left|b^{\prime}\left(v_{+}\right)\right|$but with small time-delay $0<r<\bar{r}$. Then, for any traveling waves $\phi(x+c t)$ of (1.1), whether they are monotone or nonmonotone, with the same speed $c>c_{*}$ and the same exponential decay at $\xi=-\infty$,

$$
\phi(\xi)=O(1) e^{-\lambda_{1}|\xi|} \quad \text { as } \xi \rightarrow-\infty,
$$

and they are unique up to shift.

Now we are going to state the stability result for Nicholson's birth rate case. Let $b(v)=p v e^{-a v}$; then $v_{-}=0, v_{+}=\frac{1}{a} \ln \frac{p}{d}, v_{*}=\frac{1}{a}$, and $b(v)$ automatically satisfies the conditions $\left(\mathrm{H}_{1}\right)-\left(\mathrm{H}_{3}\right)$. As a direct application of Theorem 1.2, we immediately obtain the following stability of monotone/nonmonotone traveling waves for the case with Nicholson's birth rate $b(v)=p v e^{-a v}$.

THEOREM 1.4 (stability of monotone/nonmonotone traveling waves). Let $b(v)=$ $p v e^{-a v}$. For any given traveling wave $\phi(x+c t)$ with $c>c_{*}$ connecting with $v_{-}=0$ and $v_{+}=\frac{1}{a} \ln \frac{p}{d}$, whether it is monotone or nonmonotone, suppose that $v_{0}(s, x)-$ $\phi(x+c s) \in C\left([-r, 0] ; C(R) \cap H_{w}^{2}(R)\right) \cap L^{2}\left([-r, 0] ; H_{w}^{2}(R)\right)$ and $\lim _{x \rightarrow+\infty}\left[v_{0}(s, x)-\right.$ $\phi(x+c s)]=: u_{0, \infty}(s) \in C[-r, 0]$ exists uniformly with respect to $s \in[-r, 0]$, and the initial perturbation is small:

$$
\max _{s \in[-r, 0]}\left\|\left(v_{0}-\phi\right)(s)\right\|_{C}^{2}+\left\|\left(v_{0}-\phi\right)(0)\right\|_{H_{w}^{2}}^{2}+\int_{-r}^{0}\left\|\left(v_{0}-\phi\right)(s)\right\|_{H_{w}^{2}}^{2} d s \leq \delta_{0}^{2}
$$

for some positive number $\delta_{0}>0$.

1. When $e<\frac{p}{d} \leq e^{2}$, for any time-delay $r>0$, the solution $v(t, x)$ of (1.1) and (1.2) is unique and exists globally in time in the space (1.15), and the stability (1.16) with some constant $\mu>0$ holds for all $t>0$.

2. When $\frac{p}{d}>e^{2}$ but with small time-delay $0<r<\bar{r}$, where

$$
\bar{r}:=\frac{\pi-\arctan \sqrt{\ln \frac{p}{d}\left(\ln \frac{p}{d}-2\right)}}{d \sqrt{\ln \frac{p}{d}\left(\ln \frac{p}{d}-2\right)}},
$$

the solution $v(t, x)$ of (1.1) and (1.2) also is unique and exists globally in time in the space (1.15), and the stability (1.16) with some constant $\mu>0$ holds for all $t>0$.

Remark 2.

1. In Theorem 1.4, for $e<\frac{p}{d} \leq e^{2}$ with an arbitrary time-delay $r>0$, we prove that all noncritical traveling waves with $c>c_{*}$, whether they are monotone or nonmonotone, are time-exponentially stable. Here, when $r$ is small such that $0<r<\underline{r}$, where $\underline{r}$ is defined in (1.7), the solution time-asymptotically converges to a certain monotone traveling wave, while, when $r \geq \underline{r}$, in a certain region $\mathcal{D}$ for $c$ and $r$, the solution still behaves like a monotone traveling wave. But when $c$ is really large with $c>c^{*}$ or the time-delay $r$ is large, such that $(c, r)$ is out of the region $\mathcal{D}$, then the solution behaves like a certain oscillatory traveling wave after a large time. All of these cases will be numerically demonstrated later (see Cases 1-3 in the last section of the paper).

2. When $\frac{p}{d}>e^{2}$, and the time-delay $r$ is not large, $0<r<\bar{r}$, where $\bar{r}$ is defined in (1.19), we prove that all noncritical traveling waves with $c>c_{*}$, whether they are monotone or nonmonotone, are time-exponentially stable. It can be easily verified that $0<\underline{r}<\bar{r}$ for $\frac{p}{d}>e^{2}$. Particularly, we numerically 
demonstrate in Cases 4 and 5 in section 5 that when $0<r<\underline{r}$, the solution $v(t, x)$ behaves like a monotone traveling wave, and when $\underline{r}<r<\bar{r}$, the solution $v(t, x)$ behaves like a nonmonotone traveling wave.

3. Notice that when $\frac{p}{d}>e^{2}$, as shown in [2], the sufficient and necessary condition for the stability of the solution to the linear delay differential equation (1.8) is $0<r<\bar{r}$. So, when $\frac{p}{d}>e^{2}$ and $r>\bar{r}$, we never expect any stability of traveling waves, even the traveling waves might not exist (at least, when $r \gg 1$, no traveling waves exist). In fact, from our numerical study later in Cases 6 and 7, we find that the solution doesn't behave like a traveling wave, but always oscillates chaotically at some points. Unfortunately, we are not clear about the asymptotic behavior of the solution for this challenging case.

4. When $c=c_{*}$, the approach developed in this paper cannot be applied, and the stability of critical wave $\phi\left(x+c_{*} t\right)$ remains an open problem. A new strategy will be developed in the future.

Corollary 1.5 (uniqueness of traveling waves). Let $b(v)=p v e^{-a v}$. If $e<\frac{p}{d} \leq$ $e^{2}$ with any time-delay $r>0$, or if $\frac{p}{d}>e^{2}$ but with small time-delay $0<r<\bar{r}$, where $\bar{r}$ is defined in (1.19), then, for all traveling waves $\phi(x+c t)$ to (1.1) with the same speed $c>c_{*}$ and the same exponential decay at $-\infty$, whether they are monotone or nonmonotone, these waves are unique up to shift.

Remark 3. Corollary 1.5 solves the uniqueness of monotone/nonmonotone traveling waves for $\frac{p}{d}>e$. Since uniqueness of the traveling waves for $e<\frac{p}{d} \leq e^{2}$ was proved recently by Aguerrea, Gomez, and Trofimchuk [1], here we answer the open question on the uniqueness of traveling waves for the case $\frac{p}{d} \geq e^{2}$.

The paper is outlined as follows: In section 2, we reformulate the original equation to the perturbed equation around the given traveling wave, and we give the corresponding stability theorem for the new equation. In section 3 , we consider (1.1) with a general nonmonotone birth rate function. We use the weighted energy method to establish the desired a priori estimates and use the nonlinear Halanay inequality to treat the case when $\phi(x+c t)$ nears $v_{+}$. This plays a crucial role in the proof of stability. Based on the stability theorem, in section 4 we prove the uniqueness of those monotone/nonmonotone traveling waves. Finally, in section 5, we carry out some numerical simulations which also confirm and support our theoretical results. The chaotic oscillations for the solutions in the case of $\frac{p}{d}>e^{2}$ and $r>\bar{r}$ open up a new avenue for future research.

2. Reformulation of the problem. This section is devoted to the proof of stability of those monotone or nonmonotone traveling waves. We consider (1.1) with a general nonmonotone birth rate function $b(v)$ satisfying $\left(\mathrm{H}_{1}\right)-\left(\mathrm{H}_{3}\right)$.

Let $\phi(x+c t)=\phi(\xi)$ be a given traveling wave with speed $c>c_{*}$, and

$$
u(t, \xi):=v(t, x)-\phi(x+c t), \quad u_{0}(s, \xi):=v_{0}(s, x)-\phi(x+c s) .
$$

Then, from (1.1)-(1.5), $u(t, \xi)$ satisfies

$$
\left\{\begin{array}{c}
\frac{\partial u}{\partial t}+c \frac{\partial u}{\partial \xi}-D \frac{\partial^{2} u}{\partial \xi^{2}}+d u-b^{\prime}(\phi(\xi-c r)) u(t-r, \xi-c r) \\
=Q(u(t-r, \xi-c r)), \quad(t, \xi) \in R_{+} \times R \\
u(s, \xi)=u_{0}(s, \xi), \quad s \in[-r, 0], \quad \xi \in R,
\end{array}\right.
$$

where

$$
Q(u):=b(\phi+u)-b(\phi)-b^{\prime}(\phi) u
$$


with $\phi=\phi(\xi-c r)$ and $u=u(t-r, \xi-c r)$.

Letting $T>0$, we define the solution space as

$X(-r, T)=\left\{u \mid u(t, \xi) \in C\left([-r, T] ; C(R) \cap H_{w}^{2}(R)\right) \cap L^{2}\left([-r, T] ; H_{w}^{2}(R)\right) \cap \mathcal{C}_{u n i f}[-r, T]\right\}$

equipped with the norm

$$
M_{u}(T)^{2}=\sup _{t \in[-r, T]}\left(\|u(t)\|_{C}^{2}+\|u(t)\|_{H_{w}^{2}}^{2}\right) .
$$

Particularly, when $T=\infty$, we denote the solution space by $X(-r, \infty)$ and the norm of the solution space by $M_{u}(\infty)$.

Now we state the stability result for the perturbed equation (2.1), which automatically implies Theorem 1.2.

Theorem 2.1 (stability). Let $b^{\prime}\left(v_{+}\right)$and $r$ satisfy either $d \geq\left|b^{\prime}\left(v_{+}\right)\right|$with arbitrarily given $r>0$, or $d<\left|b^{\prime}\left(v_{+}\right)\right|$with $0<r<\bar{r}$, where $\bar{r}$ is defined in (1.14). For any given traveling wave $\phi(x+c t)=\phi(\xi)$ with $c>c_{*}$, suppose that $u_{0}(s, \xi) \in C\left([-r, 0] ; C(R) \cap H_{w}^{2}(R)\right) \cap L^{2}\left([-r, 0] ; H_{w}^{2}(R)\right)$, and $\lim _{\xi \rightarrow+\infty} u_{0}(s, \xi)=:$ $u_{0, \infty}(s) \in C[-r, 0]$ exists uniformly in $s$, where $w(\xi)=e^{-2 \lambda\left(\xi-x_{0}\right)}$ with a sufficient large number $x_{0} \gg 1$. Then there exist some constants $\delta_{0}>0$ and $\mu>0$ which are independent of $u, t$, and $\xi$ when $M_{u}(0) \leq \delta_{0}$. The solution $u(t, \xi)$ of $(2.1)$ then uniquely and globally exists in $X(-r, \infty)$ and satisfies

$$
\begin{aligned}
& \|u(t)\|_{C}^{2}+\|u(t)\|_{H_{w}^{2}}^{2}+\int_{0}^{t} e^{-2 \mu(t-s)}\|u(s)\|_{H_{w}^{2}}^{2} d s \\
& \leq C e^{-2 \mu t}\left(\max _{s \in[-r, 0]}\left\|u_{0}(s)\right\|_{C}^{2}+\left\|u_{0}(0)\right\|_{H_{w}^{2}}^{2}+\int_{-r}^{0}\left\|u_{0}(s)\right\|_{H_{w}^{2}}^{2} d s\right)
\end{aligned}
$$

for $t \in[0, \infty)$.

By using the continuity extension method [27, 28], the global existence of $u(t, \xi)$ and its exponential decay estimate announced in Theorem 2.1 directly follow from the local existence result and the a priori estimate given below.

Proposition 2.2 (local existence). Under the assumption in Theorem 2.1, for any given traveling wave $\phi(x+c t)=\phi(\xi)$ with $c>c_{*}$, suppose $u_{0}(s, \xi) \in X(-r, 0)$, and $M_{u}(0) \leq \delta_{1}$ for a given positive constant $\delta_{1}>0$. Then there exists a small $t_{0}=$ $t_{0}\left(\delta_{1}\right)>0$ such that the local solution $u(t, \xi)$ of $(2.1)$ uniquely exists for $t \in\left[-r, t_{0}\right]$ and satisfies $u \in X\left(-r, t_{0}\right)$ and $M_{u}\left(t_{0}\right) \leq \bar{c}_{0} M_{u}(0)$ for some constant $\bar{c}_{0}>1$.

Proof. The proof for the local existence of the solution is standard, because it can be proved by the well-known iteration technique [21, 22]. In contrast to previous works, here we need to show that the local solution is also in $\mathcal{C}_{\text {unif }}\left[-r, t_{0}\right]$ for some small $t_{0}>0$ which will be determined later. We just sketch the proof as follows.

Let $u^{(0)}(t, \xi) \in X\left(-r, t_{0}\right)$; for example, we choose $u^{(0)}(t, \xi)=u_{0}(t, \xi)$. Then we define the iteration $u^{(n+1)}=\mathcal{P}\left(u^{(n)}\right)$ for $n \geq 0$ by

$$
\left\{\begin{array}{l}
\frac{\partial u^{(n+1)}}{\partial t}+c \frac{\partial u^{(n+1)}}{\partial \xi}-D \frac{\partial^{2} u^{(n+1)}}{\partial \xi^{2}}+d u^{(n+1)}=g\left(u^{(n)}(t-r, \xi-c r)\right), \\
u^{(n+1)}(s, \xi)=u_{0}(s, \xi), \quad s \in[-r, 0], \xi \in R
\end{array}\right.
$$

where

$$
g\left(u^{(n)}\right)=b\left(\phi+u^{(n)}\right)-b(\phi)
$$


which can be also written in the integral form

$$
\begin{aligned}
u^{(n+1)}(t, \xi)= & e^{-d t} \int_{R} G(\eta, t) u_{0}(0, \xi-\eta) d \eta \\
& +\int_{0}^{t} e^{-d(t-s)} \int_{R} G(\eta, t-s) g\left(u^{(n)}(s-r, \xi-\eta+c r)\right) d \eta d s .
\end{aligned}
$$

We also have

$$
\begin{aligned}
& e^{\mu_{2} t} u^{(n+1)}(t, \xi) \\
& =e^{-\left(d-\mu_{2}\right) t} \int_{R} G(\eta, t) u_{0}(0, \xi-\eta) d \eta \\
& \quad+\int_{0}^{t} e^{-d(t-s)} e^{\mu_{2} t} \int_{R} G(\eta, t-s) g\left(u^{(n)}(s-r, \xi-\eta+c r)\right) d \eta d s
\end{aligned}
$$

and

$$
\begin{aligned}
\partial_{\xi}^{k} u^{(n+1)}(t, \xi) \\
=e^{-d t} \int_{R} G(\eta, t) \partial_{\xi}^{k} u_{0}(0, \xi-\eta) d \eta \\
\quad+\int_{0}^{t} e^{-d(t-s)} \int_{R} G(\eta, t-s) \partial_{\xi}^{k} g\left(u^{(n)}(s-r, \xi-\eta+c r)\right) d \eta d s \\
=e^{-d t} \int_{R} \partial_{\eta}^{k} G(\eta, t) u_{0}(0, \xi-\eta) d \eta \\
\quad+\int_{0}^{t} e^{-d(t-s)} \int_{R} \partial_{\eta}^{k} G(\eta, t-s) g\left(u^{(n)}(s-r, \xi-\eta+c r)\right) d \eta d s,
\end{aligned}
$$

where $0<\mu_{2}=\mu_{2}\left(p, d, r, b^{\prime}\left(v_{+}\right)\right)<d$ is a decay exponent for the delayed ODE (3.21) and specified in Lemma 3.10, and $G(\eta, t)$ is the heat kernel

$$
G(\eta, t)=\frac{1}{\sqrt{4 \pi D t}} e^{-\frac{(\eta+c t)^{2}}{4 D t}}
$$

Notice that $u^{(n)} \in \mathcal{C}_{u n i f}\left[-r, t_{0}\right]$, namely, $\lim _{\xi \rightarrow \infty} e^{\mu_{2} t} u^{(n)}(t, \xi)=: e^{\mu_{2} t} u_{\infty}^{(n)}(t) \in C\left[-r, t_{0}\right]$ and $\lim _{\xi \rightarrow \infty} \partial_{\xi}^{k} u^{(n)}(t, \xi)=0$ all exist uniformly in $t$ for $k=1$, 2; we are going to prove $u^{(n+1)} \in \mathcal{C}_{\text {unif }}\left[-r, t_{0}\right]$. First of all, we note that

$$
\begin{aligned}
\lim _{\xi \rightarrow \infty} u^{(n+1)}(t, \xi) \\
=e^{-d t} \int_{R} G(\eta, t) \lim _{\xi \rightarrow \infty} u_{0}(0, \xi-\eta) d \eta \\
+\int_{0}^{t} e^{-d(t-s)} \int_{R} G(\eta, t-s) \lim _{\xi \rightarrow \infty} g\left(u^{(n)}(s-r, \xi-\eta+c r)\right) d \eta d s \\
=u_{0, \infty}(0) e^{-d t} \int_{R} G(\eta, t) d \eta \\
+\int_{0}^{t} e^{-d(t-s)} g\left(u_{\infty}^{(n)}(s-r)\right) \int_{R} G(\eta, t-s) d \eta d s \\
=u_{0, \infty}(0) e^{-d t}+\int_{0}^{t} e^{-d(t-s)} g\left(u_{\infty}^{(n)}(s-r)\right) d s \\
=: u_{\infty}^{(n+1)}(t), \quad \text { uniformly with respect to } t \in\left[-r, t_{0}\right] .
\end{aligned}
$$


Now we prove that $e^{\mu_{2} t} u^{(n+1)}(t, \xi)$ is uniformly convergent as $\xi \rightarrow \infty$. In fact,

$$
\begin{aligned}
& \lim _{\xi \rightarrow \infty} \sup _{0 \leq t \leq t_{0}}\left|e^{\mu_{2} t} u^{(n+1)}(t, \xi)-e^{\mu_{2} t} u_{\infty}^{(n+1)}(t)\right| \\
&=\lim _{\xi \rightarrow \infty} \sup _{0 \leq t \leq t_{0}} \int_{0}^{t} e^{-d(t-s)} e^{\mu_{2}(t-s+r)} \\
& \quad \times \int_{R} G(\eta, t-s) e^{\mu_{2}(s-r)}\left[g\left(u^{(n)}(s-r, \xi-\eta+c r)\right)-g\left(u_{\infty}^{(n)}(s-r)\right)\right] d \eta d s \\
& \leq \lim _{\xi \rightarrow \infty} \sup _{0 \leq t \leq t_{0}} \int_{0}^{t} e^{-d(t-s)} e^{\mu_{2}(t-s+r)} \\
& \quad \times \int_{R} G(\eta, t-s) \sup _{s \in\left[0, t_{0}\right]}\left(e^{\mu_{2}(s-r)}\left|g\left(u^{(n)}(s-r, \xi-\eta+c r)\right)-g\left(u_{\infty}^{(n)}(s-r)\right)\right|\right) d \eta d s \\
& \leq C \lim _{\xi \rightarrow \infty} \sup _{0 \leq t \leq t_{0}} \int_{0}^{t} e^{-d(t-s)} e^{\mu_{2}(t-s+r)} \\
& \times \int_{R} G(\eta, t-s) \sup _{s \in\left[0, t_{0}\right]}\left(e^{\mu_{2}(s-r)}\left|u^{(n)}(s-r, \xi-\eta+c r)-u_{\infty}^{(n)}(s-r)\right|\right) d \eta d s \\
&= C \sup _{0 \leq t \leq t_{0}} \int_{0}^{t} e^{-d(t-s)} e^{\mu_{2}(t-s+r)} \\
& \times \int_{R} G(\eta, t-s) \lim _{\xi \rightarrow \infty} \sup _{s \in\left[0, t_{0}\right]}\left(e^{\mu_{2}(s-r)}\left|u^{(n)}(s-r, \xi-\eta+c r)-u_{\infty}^{(n)}(s-r)\right|\right) d \eta d s \\
&= 0 .
\end{aligned}
$$

Here we used the uniform convergence of

$$
\lim _{\xi \rightarrow \infty} \sup _{t \in\left[0, t_{0}\right]}\left|e^{\mu_{2} t} u^{(n)}(t, \xi)-e^{\mu_{2} t} u_{\infty}^{(n)}(t)\right|=0
$$

and the uniform boundedness of

$$
\int_{0}^{t} e^{-d(t-s)} e^{\mu_{2}(t-s+r)} d s=\frac{e^{\mu_{2} r}}{d-\mu_{2}}\left[1-e^{-\left(d-\mu_{2}\right) t}\right] \leq \frac{e^{\mu_{2} r}}{d-\mu_{2}}
$$

and

$$
\int_{R} G(\eta, t-s) d \eta=1
$$

Similarly, noting the facts

$$
\left.G(\eta, t)\right|_{\eta= \pm \infty}=0 \text { and }\left.\left(\partial_{\eta} G(\eta, t)\right)\right|_{\eta= \pm \infty}=0,
$$

we can prove that, for $k=1,2$,

$$
\begin{aligned}
\lim _{\xi \rightarrow \infty} \partial_{\xi}^{k} u^{(n+1)}(t, \xi) \\
=e^{-d t} \int_{R} \partial_{\eta}^{k} G(\eta, t) \lim _{\xi \rightarrow \infty} u_{0}(0, \xi-\eta) d \eta \\
+\int_{0}^{t} e^{-d(t-s)} \int_{R} \partial_{\eta}^{k} G(\eta, t-s) \lim _{\xi \rightarrow \infty} g\left(u^{(n)}(s-r, \xi-\eta+c r)\right) d \eta d s \\
=u_{0, \infty}(0) e^{-d t} \int_{R} \partial_{\eta}^{k} G(\eta, t) d \eta
\end{aligned}
$$




$$
+\int_{0}^{t} e^{-d(t-s)} g\left(u_{\infty}^{(n)}(s-r)\right) \int_{R} \partial_{\eta}^{k} G(\eta, t-s) d \eta d s
$$

$=0, \quad$ uniformly with respect to $t \in\left[-r, t_{0}\right]$.

Furthermore, by taking the regular energy estimates

$$
\int_{0}^{t} \int_{R}\left(\sum_{k=0}^{2} \partial_{\xi}^{k}(2.6) \times w(\xi) \partial_{\xi}^{k} u^{(n+1)}\right) d \xi d s,
$$

we can estimate

$$
\begin{aligned}
& \left\|u^{(n+1)}(t)\right\|_{H_{w}^{2}}^{2}+\int_{0}^{t}\left\|u^{(n+1)}(s)\right\|_{H_{w}^{2}}^{2} d s \\
& \leq C\left(\left\|u_{0}(0)\right\|_{H_{w}^{2}}^{2}+\int_{-r}^{0}\left\|u_{0}(s)\right\|_{H_{w}^{2}}^{2} d s+\int_{0}^{t}\left\|u^{(n)}(s)\right\|_{H_{w}^{2}}^{2} d s\right), \quad t \in\left[0, t_{0}\right],
\end{aligned}
$$

for some positive constant $C>0$. From (2.7), we have

$$
\left\|u^{(n+1)}(t)\right\|_{C} \leq C\left\|u_{0}(0)\right\|_{C}+C t_{0} \sup _{t \in\left[-r, t_{0}\right]}\left\|u^{(n)}(t)\right\|_{C}, \quad t \in\left[0, t_{0}\right] .
$$

Combining (2.10) and (2.11), we prove

$$
\begin{aligned}
M_{u^{(n+1)}}\left(t_{0}\right) \leq & C\left(\max _{s \in[-r, 0]}\left\|u_{0}(s)\right\|_{C}^{2}+\left\|u_{0}(0)\right\|_{H_{w}^{2}}^{2}+\int_{-r}^{0}\left\|u_{0}(s)\right\|_{H_{w}^{2}}^{2} d s\right) \\
& +C t_{0} M_{u^{(n)}}\left(t_{0}\right) .
\end{aligned}
$$

Thus, we can prove that $u^{(n+1)}=\mathcal{P}\left(u^{(n)}\right)$ defined in (2.6) maps from $X\left(-r, t_{0}\right)$ to $X\left(-r, t_{0}\right)$ and is a contraction mapping in $X\left(-r, t_{0}\right)$ by providing $0<t_{0} \ll 1$ and $\max _{s \in[-r, 0]}\left\|u_{0}(s)\right\|_{C}^{2}+\left\|u_{0}(0)\right\|_{H_{w}^{2}}^{2}+\int_{-r}^{0}\left\|u_{0}(s)\right\|_{H_{w}^{2}}^{2} d s \ll 1$. Hence, by applying the Banach fixed point theorem, we can prove local existence of the solution in $X\left(-r, t_{0}\right)$. Since the convergence $\lim _{n \rightarrow \infty} u^{(n)}(t, \xi)=u(t, \xi)$ is uniform for $(t, \xi) \in\left[0, t_{0}\right] \times R$, and $u^{(n)} \in \mathcal{C}_{\text {unif }}\left[0, t_{0}\right]$, we can also guarantee $u \in \mathcal{C}_{\text {unif }}\left[0, t_{0}\right]$.

Proposition 2.3 (a priori estimates). Under the assumption in Theorem 2.1, let $u \in X(-r, T)$ be a local solution of (2.1) for a given constant $T>0$. Then there exist positive constants $\delta_{2}>0, C_{0}>1$, and $\mu>0$ independent of $T$ and $u(t, \xi)$ such that, when $M_{u}(T) \leq \delta_{2}$,

$$
\begin{aligned}
& \|u(t)\|_{C}^{2}+\|u(t)\|_{H_{w}^{1}}^{2}+\int_{0}^{t} e^{-2 \mu(t-s)}\|u(s)\|_{H_{w}^{1}}^{2} d s \\
& \leq C_{0} e^{-2 \mu t}\left(\max _{s \in[-r, 0]}\left\|u_{0}(s)\right\|_{L^{\infty}}^{2}+\left\|u_{0}(0)\right\|_{H_{w}^{1}}^{2}+\int_{-r}^{0}\left\|u_{0}(s)\right\|_{H_{w}^{1}}^{2} d s\right)
\end{aligned}
$$

for $t \in[0, T]$.

The proof for the a priori estimates of the solution in the designed solution space $X(-r, T)$ is technical and plays a crucial role in this paper. We leave this for the next section.

Proof of Theorem 2.1. Since this can be proved similarly as done in [27, 28], here we sketch the proof as follows. Let $\delta_{2}, C_{0}$, and $\mu$ be the positive constants given in Proposition 2.3 which are independent of $T$ and $u$. Now let us choose

$$
\delta_{1}=\max \left\{\sqrt{C_{0}} \bar{c}_{0} M_{u}(0), \delta_{2}\right\}, \quad \delta_{0}=\max \left\{\frac{\delta_{2}}{\overline{\bar{c}}_{0}}, \frac{\delta_{2}}{\sqrt{C_{0}} \bar{c}_{0}}\right\}
$$


By Proposition 2.2, there exists $t_{0}=t_{0}\left(\delta_{1}\right)>0$ such that $u(t, \xi) \in X\left(-r, t_{0}\right)$. From the selection of $\delta_{0}$ and $\delta_{1}$, we can further confirm $M_{u}\left(t_{0}\right) \leq \delta_{2}$. Then, applying Proposition 2.3, we can establish the exponential decay estimate (2.12) for $t \in\left[0, t_{0}\right]$. Next, let us consider $(2.1)$ with the new initial data $u(s, \xi)$ for $s \in\left[t_{0}-r, t_{0}\right]$. Again, by Proposition 2.2, we can prove that the solution to the new Cauchy problem (2.1) exists for time $t$ in $\left[t_{0}, 2 t_{0}\right]$; namely, we extend the time interval of the solution to $\left[-r, 2 t_{0}\right]$, or say, $u(t, \xi) \in X\left(-r, 2 t_{0}\right)$. Then, by using Proposition 2.3, we can establish the exponential decay estimate $(2.12)$ for $t \in\left[0,2 t_{0}\right]$. Repeating this procedure, we can prove global existence of the solution $u(t, \xi) \in X(-r, \infty)$ with the exponential decay estimate $(2.12)$ for $t \in[0, \infty)$. For details, we refer the reader to [27].

3. A priori estimates. This section is devoted to establishing the a priori estimates, which are the core of the paper. The adopted approach is still the weighted energy method but with a new development. Here, the birth rate function $b(v)$ is, in general, considered as an unimodality function satisfying $\left(\mathrm{H}_{1}\right)-\left(\mathrm{H}_{3}\right)$.

First of all, we are going to establish the energy estimates for $u(t, \xi)$ in the weighted Sobolev space $H_{w}^{2}(R)$.

Lemma 3.1. Let $u(t, \xi) \in X(-r, T)$. Then

$$
\begin{aligned}
& \|u(t)\|_{L_{w}^{2}}^{2}+\int_{0}^{t} e^{-2 \mu(t-s)} \int_{R}\left[\mathcal{B}_{\eta, \mu, w}(\xi)-C M_{u}(T)\right] w(\xi) u^{2}(s, \xi) d \xi d s \\
& \leq C e^{-2 \mu t}\left(\left\|u_{0}(0)\right\|_{L_{w}^{2}}^{2}+\int_{-r}^{0}\left\|u_{0}(s)\right\|_{L_{w}^{2}}^{2} d s\right),
\end{aligned}
$$

where

$$
\mathcal{B}_{\eta, \mu, w}(\xi):=\mathcal{A}_{\eta, w}(\xi)-2 \mu-\frac{1}{\eta}\left(e^{2 \mu r}-1\right)\left|b^{\prime}(\phi(\xi))\right| \frac{w(\xi+c r)}{w(\xi)}
$$

and

$$
\mathcal{A}_{\eta, w}(\xi):=-c \frac{w^{\prime}(\xi)}{w(\xi)}+2 d-\frac{D}{2}\left(\frac{w^{\prime}(\xi)}{w(\xi)}\right)^{2}-\eta\left|b^{\prime}(\phi(\xi-c r))\right|-\frac{1}{\eta}\left|b^{\prime}(\phi(\xi))\right| \frac{w(\xi+c r)}{w(\xi)}
$$

and $\mu$ and $\eta$ both are arbitrarily given positive constants at this moment, but will be specified later.

Proof. As shown in our previous work [23], we multiply (2.1) by $e^{2 \mu t} w(\xi) u(t, \xi)$, where $\mu>0$ is a constant and will be specified later in Lemma 3.3. Then we have

$$
\begin{aligned}
& \left\{\frac{1}{2} e^{2 \mu t} w u^{2}\right\}_{t}+e^{2 \mu t}\left\{\frac{1}{2} c w u^{2}-D w u u_{\xi}\right\}_{\xi}+D e^{2 \mu t} w u_{\xi}^{2}+D e^{2 \mu t} w^{\prime} u_{\xi} u \\
& \quad+\left\{-\frac{c}{2} \frac{w^{\prime}}{w}+d-\mu\right\} e^{2 \mu t} w u^{2}-e^{2 \mu t} w(\xi) u(t, \xi) b^{\prime}(\phi(\xi-c r)) u(t-r, \xi-c r)
\end{aligned}
$$

By the Cauchy-Schwarz inequality

$$
\left|D e^{2 \mu t} w^{\prime} u u_{\xi}\right| \leq D e^{2 \mu t} w u_{\xi}^{2}+\frac{D}{4} e^{2 \mu t}\left(\frac{w^{\prime}}{w}\right)^{2} w u^{2},
$$

we reduce (3.4) to

$$
\left\{\frac{1}{2} e^{2 \mu t} w u^{2}\right\}_{t}+e^{2 \mu t}\left\{\frac{1}{2} c w u^{2}-D w u u_{\xi}\right\}_{\xi}
$$




$$
\begin{aligned}
& \quad+\left\{-\frac{c}{2} \frac{w^{\prime}}{w}+d-\mu-\frac{D}{4}\left(\frac{w^{\prime}}{w}\right)^{2}\right\} e^{2 \mu t} w u^{2} \\
& \quad-e^{2 \mu t} w(\xi) u(t, \xi) b^{\prime}(\phi(\xi-c r)) u(t-r, \xi-c r) \\
& \leq e^{2 \mu t} w(\xi) u(t, \xi) Q(u(t-r, \xi-c r)) .
\end{aligned}
$$

Integrating the above inequality over $R \times[0, t]$ with respect to $\xi$ and $t$, and noting the vanishing term at far fields,

$$
\left.\left\{\frac{1}{2} c w u^{2}-D w u u_{\xi}\right\}\right|_{\xi=-\infty} ^{\infty}=0
$$

because $\sqrt{w} u \in H^{2}(R)$ (see Lemma 3.7 below), this implies, by the property of Sobolev space $H^{2}(R)$, that $\left.(\sqrt{w} u)\right|_{\xi= \pm \infty}=0$ and $\left.\left(\sqrt{w} u_{\xi}\right)\right|_{\xi= \pm \infty}=0$. Thus we further have

$$
\begin{aligned}
& e^{2 \mu t}\|u(t)\|_{L_{w}^{2}}^{2}+\int_{0}^{t} \int_{R} e^{2 \mu s}\left\{-c \frac{w^{\prime}(\xi)}{w(\xi)}+2 d-2 \mu-\frac{D}{2}\left(\frac{w^{\prime}(\xi)}{w(\xi)}\right)^{2}\right\} w(\xi) u^{2}(s, \xi) d \xi d s \\
& -2 \int_{0}^{t} \int_{R} e^{2 \mu s} w(\xi) b^{\prime}(\phi(\xi-c r)) u(s, \xi) u(s-r, \xi-c r) d \xi d s \\
& \leq\left\|u_{0}(0)\right\|_{L_{w}^{2}}^{2}+2 \int_{0}^{t} \int_{R} e^{2 \mu s} w(\xi) u(s, \xi) Q(u(s-r, \xi-c r)) d \xi d s .
\end{aligned}
$$

Again, by using the Cauchy-Schwarz inequality

$$
\begin{aligned}
& \left|2 e^{2 \mu s} w(\xi) b^{\prime}(\phi(\xi-c r)) u(s, \xi) u(s-r, \xi-c r)\right| \\
& \leq e^{2 \mu s} w(\xi)\left|b^{\prime}(\phi(\xi-c r))\right|\left[\eta u^{2}(s, \xi)+\frac{1}{\eta} u^{2}(s-r, \xi-c r)\right]
\end{aligned}
$$

for any $\eta>0$, and by change of variables, we have

$$
\begin{aligned}
\mid & 2 \int_{0}^{t} \int_{R} e^{2 \mu s} w(\xi) b^{\prime}(\phi(\xi-c r)) u(s, \xi) u(s-r, \xi-c r) d \xi d s \mid \\
\leq & \eta \int_{0}^{t} \int_{R} e^{2 \mu s} w(\xi)\left|b^{\prime}(\phi(\xi-c r))\right| u^{2}(s, \xi) d \xi d s \\
& +\frac{1}{\eta} \int_{0}^{t} \int_{R} e^{2 \mu s} w(\xi)\left|b^{\prime}(\phi(\xi-c r))\right| u^{2}(s-r, \xi-c r) d \xi d s \\
= & \eta \int_{0}^{t} \int_{R} e^{2 \mu s} w(\xi)\left|b^{\prime}(\phi(\xi-c r))\right| u^{2}(s, \xi) d \xi d s \\
& +\frac{1}{\eta} e^{2 \mu r} \int_{-r}^{t-r} \int_{R} e^{2 \mu s} w(\xi+c r)\left|b^{\prime}(\phi(\xi))\right| u^{2}(s, \xi) d \xi d s \\
\leq & \quad[\operatorname{by} \operatorname{setting} \xi-c r \rightarrow \xi, \quad s-r \rightarrow s] \\
& +\frac{1}{\eta} e_{0}^{t} \int_{R} e^{2 \mu r} \int_{0}^{t} \int_{R} e^{2 \mu s} w(\xi+c r)\left|b^{\prime}(\phi(\xi))\right| u^{2}(s, \xi) d \xi d s \\
& +\frac{1}{\eta} e^{2 \mu r} \int_{-r}^{0} \int_{R} e^{2 \mu s} w(\xi+c r)\left|b^{\prime}(\phi(\xi))\right| u_{0}^{2}(s, \xi) d \xi d s .
\end{aligned}
$$


Substituting (3.7) into (3.6), we have

$$
\begin{aligned}
& e^{2 \mu t}\|u(t)\|_{L_{w}^{2}}^{2}+\int_{0}^{t} \int_{R} e^{2 \mu s} \mathcal{B}_{\eta, \mu, w}(\xi) w(\xi) u^{2}(s, \xi) d \xi d s \\
& \leq\left\|u_{0}(0)\right\|_{L_{w}^{2}}^{2}+2 \int_{0}^{t} \int_{R} e^{2 \mu s} w(\xi) u(s, \xi) Q(u(s-r, \xi-c r)) d \xi d s \\
& +\eta^{-1} e^{2 \mu r} \int_{-r}^{0} \int_{R} e^{2 \mu s} w(\xi+c r)\left|b^{\prime}(\phi(\xi))\right| u_{0}^{2}(s, \xi) d \xi d s,
\end{aligned}
$$

where $\mathcal{B}_{\eta, \mu, w}(\xi)$ is given in (3.2).

On the other hand, by the definition of $M_{u}(T)$ (see (2.4)),

$$
|u(t, \xi)| \leq C M_{u}(T) \quad \text { for } t \in[0, T], \xi \in R,
$$

and by the Taylor expansion

$$
|Q(u)|=\left|b(u+\phi)-b(\phi)-b^{\prime}(\phi) u\right| \leq C|u|^{2},
$$

where $C>0$ is independent of $u$, we can estimate the nonlinear term as

$$
\begin{aligned}
& \left|\int_{0}^{t} \int_{R} e^{2 \mu s} w(\xi) u(s, \xi) Q(u(s-r, \xi-c r)) d \xi d s\right| \\
& \leq C M_{u}(T) \int_{0}^{t} \int_{R} e^{2 \mu s} w(\xi)|u(s-r, \xi-c r)|^{2} d \xi d s \\
& =C M_{u}(T) \int_{-r}^{t-r} \int_{R} e^{2 \mu(s+r)} w(\xi)|u(s, \xi)|^{2} d \xi d s \\
& \leq C M_{u}(T) \int_{0}^{t} \int_{R} e^{2 \mu s} w(\xi)|u(s, \xi)|^{2} d \xi d s \\
& \quad+C M_{u}(T) \int_{-r}^{0} \int_{R} e^{2 \mu s} w(\xi+c r)\left|u_{0}(s, \xi)\right|^{2} d \xi d s .
\end{aligned}
$$

Here, the last estimate can be followed from (3.7), and $w(\xi+c r)=e^{-2 \lambda c r} w(\xi)$. Substituting (3.9) into (3.8), we have

$$
\begin{aligned}
& e^{2 \mu t}\|u(t)\|_{L_{w}^{2}}^{2}+\int_{0}^{t} e^{2 \mu s} \int_{R}\left[\mathcal{B}_{\eta, \mu, w}(\xi)-C M_{u}(T)\right] w(\xi) u^{2}(s, \xi) d \xi d s \\
& \leq C\left(\left\|u_{0}(0)\right\|_{L_{w}^{2}}^{2}+\int_{-r}^{0}\left\|u_{0}(s)\right\|_{L_{w}^{2}}^{2} d s\right)
\end{aligned}
$$

which immediately implies (3.1). Thus, the proof is complete.

LEMmA 3.2. Let $\eta=e^{-\lambda c r}$. Then there exists a constant $C_{1}>0$ such that

$$
\mathcal{A}_{\eta, w}(\xi) \geq C_{1}>0 \quad \text { for } \xi \in R .
$$

Proof. By $\left(\mathrm{H}_{3}\right)$, notice that

$$
\left|b^{\prime}(\phi)\right| \leq b^{\prime}(0) \text { for all } \phi \geq 0
$$

and

$$
\frac{w^{\prime}(\xi)}{w(\xi)}=-2 \lambda, \quad \frac{w(\xi+c r)}{w(\xi)}=e^{-2 \lambda c r}
$$


Then by using the fact (1.11), i.e.,

$$
c \lambda+d-D \lambda^{2}-b^{\prime}(0) e^{-\lambda c r}>0 \quad \text { for } c>c_{*} \text { and } \lambda \in\left(\lambda_{1}, \lambda_{2}\right),
$$

we can guarantee that $\mathcal{A}_{\eta, w}(\xi)$ as defined in (3.3) is always positive, that is,

$$
\begin{aligned}
& \mathcal{A}_{\eta, w}(\xi)=-c \frac{w^{\prime}(\xi)}{w(\xi)}+2 d-\frac{D}{2}\left(\frac{w^{\prime}(\xi)}{w(\xi)}\right)^{2} \\
&-e^{-\lambda c r}\left|b^{\prime}(\phi(\xi-c r))\right|-e^{\lambda c r} \frac{w(\xi+c r)}{w(\xi)}\left|b^{\prime}(\phi(\xi))\right| \\
& \geq 2\left[c \lambda+d-D \lambda^{2}-b^{\prime}(0) e^{-\lambda c r}\right] \\
&=: C_{1}>0
\end{aligned}
$$

for $\xi \in R$ with $c>c_{*}$ and $\lambda \in\left(\lambda_{1}, \lambda_{2}\right)$.

Remark 4. The choice of $\eta$ and the weight function $w(\xi)$ are also delicate and important, because with a different setting for $\eta$ and $w(\xi)$, we may not be able to get the positivity (3.11) of $\mathcal{A}_{\eta, w}(\xi)$ for every speed $c>c_{*}$. Otherwise, as in $[28,27,18$, $39,40]$, we need to restrict the speed to be large, $c \gg c_{*}$. It is worth pointing out that such a technical selection of $\eta=e^{\lambda c r}$ was first given by Gourley in [9] on the linear stability of wavefronts for an age-structured population model.

Lemma 3.3. Let $u(t, \xi) \in X(-r, T)$. Then there exists a constant $\mu_{1}>0$ such that, for $0<\mu<\mu_{1}$, it holds that

$$
\|u(t)\|_{L_{w}^{2}}^{2}+\int_{0}^{t} e^{-2 \mu(t-s)}\|u(s)\|_{L_{w}^{2}}^{2} d s \leq C e^{-2 \mu t}\left(\left\|u_{0}(0)\right\|_{L_{w}^{2}}^{2}+\int_{-r}^{0}\left\|u_{0}(s)\right\|_{L_{w}^{2}}^{2} d s\right),
$$

provided $M_{u}(T) \ll 1$.

Proof. By (3.10), we estimate $\mathcal{B}_{\eta, \mu, w}(\xi)$ as defined in (3.2) as

$$
\begin{aligned}
\mathcal{B}_{\eta, \mu, w}(\xi) & =\mathcal{A}_{\eta, w}(\xi)-2 \mu-e^{\lambda c r}\left(e^{2 \mu r}-1\right)\left|b^{\prime}(\phi(\xi))\right| \frac{w(\xi+c r)}{w(\xi)} \\
& \geq C_{1}-2 \mu-b^{\prime}(0) e^{-\lambda c r}\left(e^{2 \mu r}-1\right) \\
& =: C_{2}>0
\end{aligned}
$$

by selecting $\mu$ to be small enough such that

$$
0<\mu \leq \mu_{1}
$$

where $\mu_{1}>0$ is the unique positive solution of the equation

$$
C_{1}-2 \mu_{1}-b^{\prime}(0) e^{-\lambda c r}\left(e^{2 \mu_{1} r}-1\right)=0 .
$$

Applying (3.13) to (3.1), we get

$$
\begin{aligned}
& \|u(t)\|_{L_{w}^{2}}^{2}+\int_{0}^{t} e^{-2 \mu(t-s)} \int_{R}\left[C_{2}-C M_{u}(T)\right] w(\xi) u^{2}(s, \xi) d \xi d s \\
& \leq C e^{-2 \mu t}\left(\left\|u_{0}(0)\right\|_{L_{w}^{2}}^{2}+\int_{-r}^{0}\left\|u_{0}(s)\right\|_{L_{w}^{2}}^{2} d s\right)
\end{aligned}
$$

which then implies $(3.12)$ by letting $M_{u}(T) \ll 1$. Thus, the proof of this lemma is complete. 
Next we derive the estimates for the higher order derivatives of the solution.

Lemma 3.4. Let $u(t, \xi) \in X(-r, T)$. Then it holds that

$$
\begin{aligned}
& \left\|u_{\xi}(t)\right\|_{L_{w}^{2}}^{2}+\int_{0}^{t} e^{-2 \mu(t-s)}\left\|u_{\xi}(s)\right\|_{L_{w}^{2}}^{2} d s \\
& \leq C e^{-2 \mu t}\left(\left\|u_{0}(0)\right\|_{H_{w}^{1}}^{2}+\int_{-r}^{0}\left\|u_{0}(s)\right\|_{H_{w}^{1}}^{2} d s\right),
\end{aligned}
$$

provided $M_{u}(T) \ll 1$.

Proof. Differentiating (2.1) with respect to $\xi$ and multiplying it by $e^{2 \mu t} w(\xi) u_{\xi}(t, \xi)$, then integrating the resultant equation with respect to $\xi$ and $t$ over $R \times[0, t]$ and applying Lemma 3.3, we can similarly prove (3.16). The details are omitted.

Similarly, by taking

$$
\int_{0}^{t} \int_{R} \partial_{\xi}^{2}(2.1) \times w(\xi) \partial_{\xi}^{2} u d \xi d s
$$

and applying Lemmas 3.3 and 3.4, we obtain the energy estimates for $u_{\xi \xi}$.

Lemma 3.5. Let $u(t, \xi) \in X(-r, T)$. Then it holds that

$$
\begin{aligned}
& \left\|u_{\xi \xi}(t)\right\|_{L_{w}^{2}}^{2}+\int_{0}^{t} e^{-2 \mu(t-s)}\left\|u_{\xi \xi}(s)\right\|_{L_{w}^{2}}^{2} d s \\
& \leq C e^{-2 \mu t}\left(\left\|u_{0}(0)\right\|_{H_{w}^{2}}^{2}+\int_{-r}^{0}\left\|u_{0}(s)\right\|_{H_{w}^{2}}^{2} d s\right),
\end{aligned}
$$

provided $M_{u}(T) \ll 1$.

Remark 5. In order to establish the energy estimate (3.16), technically we need a good enough regularity for the solution $u(t, \xi)$. To do this, the usual approach is via the mollification $[22,25]$, e.g., let us mollify the initial data such that it can be in $C^{\infty}$, which then ensures the solution $u$ will have good enough regularity. Thus, the approach taken in the proof of Lemma 3.4 is applicable. Here, for the sake of simplicity, we omit the details. Actually, the same mollification procedure is also needed in the proof of Lemma 3.1, but we ignore it for the same reason.

Combining Lemmas 3.3 and 3.4, we prove the following a priori estimates.

Lemma 3.6. Let $u(t, \xi) \in X(-r, T)$. Then

$$
\begin{aligned}
& \|u(t)\|_{H_{w}^{2}}^{2}+\int_{0}^{t} e^{-2 \mu(t-s)}\|u(s)\|_{H_{w}^{2}}^{2} d s \\
& \leq C e^{-2 \mu t}\left(\left\|u_{0}(0)\right\|_{H_{w}^{2}}^{2}+\int_{-r}^{0}\left\|u_{0}(s)\right\|_{H_{w}^{2}}^{2} d s\right),
\end{aligned}
$$

provided $M_{u}(T) \ll 1$.

Next, we establish the following Sobolev inequality.

Lemma 3.7. Let $u \in H_{w}^{2}(R)$. Then it is equivalent to $\sqrt{w} u \in H^{2}(R)$ and

$$
\|\sqrt{w} u\|_{C^{1}} \leq C\|u\|_{H_{w}^{2}}
$$

and

$$
\sup _{\xi \in\left(-\infty, x_{0}\right]}|u(t, \xi)| \leq C \delta_{0} e^{-\mu t}, \quad t>0 .
$$


Proof. Since $u \in H_{w}^{2}(R)$, i.e., $\sqrt{w} u \in L^{2}(R), \sqrt{w} u_{\xi} \in L^{2}(R)$, and $\sqrt{w} u_{\xi \xi} \in$ $L^{2}(R)$, it immediately yields $\partial_{\xi}(\sqrt{w} u)=\sqrt{w} u_{\xi}-\lambda \sqrt{w} u \in L^{2}(R)$ and $\partial_{\xi \xi}(\sqrt{w} u)=$ $\sqrt{w} u_{\xi \xi}-2 \lambda \sqrt{w} u_{\xi}+\lambda^{2} \sqrt{w} u \in L^{2}(R)$. So, we prove that $\sqrt{w} u \in H^{2}(R)$. Thus, by using the Sobolev inequality $H^{2} \hookrightarrow C^{1}$, we get $\|\sqrt{w} u\|_{C^{1}} \leq C\|u\|_{H_{w}^{2}}$, which implies, from (3.18) and the fact $w(\xi)=e^{-2 \lambda\left(\xi-x_{0}\right)} \geq 1$ for $\xi \in\left(-\infty, x_{0}\right]$, that

$$
\sup _{\xi \in\left(-\infty, x_{0}\right]}|u(t, \xi)| \leq C \delta_{0} e^{-\mu t}, \quad t>0 .
$$

This completes the proof.

Next, let us investigate the time-exponential decay of $u(t, \xi)$ at $\xi=+\infty$. Let $u(t, \xi) \in X(-r, T)$. By the definition of $\mathcal{C}_{\text {unif }}[0, T]$, we have that $\lim _{\xi \rightarrow+\infty} u(t, \xi)$ exists uniformly with respect to $t \in[0, T]$, and $\lim _{\xi \rightarrow+\infty} u_{\xi}(t, \xi)=\lim _{\xi \rightarrow+\infty} u_{\xi \xi}(t, \xi)=0$ uniformly with respect to $t \in[0, T]$. Denote $z(t):=u(t, \infty)$ and $z_{0}(s):=u_{0}(s, \infty)$ for $s \in[-r, 0]$. By taking $\xi \rightarrow \infty$ to (2.1), we have

$$
\left\{\begin{array}{l}
z^{\prime}(t)+d z(t)-b^{\prime}\left(v_{+}\right) z(t-r)=Q(z(t-r)), \\
z(s)=z_{0}(s), s \in[-r, 0] .
\end{array}\right.
$$

In order to estimate this nonlinear delayed ODE, let us recall the exponential decay of the solution for the following linear equation in a general form:

$$
\left\{\begin{array}{l}
z^{\prime}(t)+k_{1} z(t)=k_{2} z(t-r), \\
z(s)=z_{0}(s), \quad s \in[-r, 0],
\end{array}\right.
$$

where $k_{1}$ and $k_{2}$ are arbitrarily given constants.

LEMmA 3.8 (see $[2,8,13,15,33,36]$ ).

(i) When $0<k_{2}<k_{1}$, then, for any time-delay $r>0$, it holds that

$$
|z(t)| \leq M e^{-\varepsilon_{1}\left(k_{1}-k_{2}\right) t}, \quad t>0,
$$

for some positive constant $0<\varepsilon_{1}<1$, where $M$ is chosen as

$$
M \geq \max _{s \in[-r, 0]}\left|z_{0}(s)\right| .
$$

(ii) When $0<k_{1} \leq k_{2}$, then, for any time-delay $r>0$, it diverges as

$$
|z(t)|=O(1) e^{\nu t}, \quad t>0,
$$

where $\nu>0$ is uniquely determined by

$$
\nu+k_{1}=k_{2} e^{-\nu r},
$$

which implies

$$
0<\nu<\frac{1}{r}\left(\ln k_{2}-\ln k_{1}\right) .
$$

(iii) When $k_{2}<0<k_{1}$ and $\left|k_{2}\right| \leq k_{1}$, then, for any time-delay $r>0$, it holds that

$$
|z(t)| \leq C e^{-\nu t}, \quad t>0,
$$

for some $\nu>0$. 
(iv) When $k_{2}<0<k_{1},\left|k_{2}\right|>k_{1}$, then it holds that

$$
|z(t)| \leq C e^{-\nu t}, \quad t>0,
$$

for some $\nu>0$ if and only if $0<r<\bar{r}$, where

$$
\bar{r}:=\left[\pi-\arctan \left(k_{1}^{-1} \sqrt{\left|k_{2}\right|^{2}-k_{1}^{2}}\right)\right] / \sqrt{\left|k_{2}\right|^{2}-k_{1}^{2}} .
$$

(v) Here, for $k_{2}<0<k_{1}$, when time-delay $r>\underline{r}$, where $\underline{r}>0$ is defined by

$$
\left|k_{2}\right| \underline{r} e^{k_{1} \underline{r}+1}=1
$$

then all solutions $z(t)$ of (3.22) are always oscillatory.

The proof is standard. Here, (i)-(iii) are immediately derived from the textbooks $[13,15,33]$, (iv) is originally from the stability analysis for linear delay differential equations by Boese [2] (see also the summery in the textbook [13]), and the oscillation part (v) is a simple corollary of [36] (see also the textbook [8]).

Next, let us consider a nonlinear perturbation to the linear delay differential equation (3.22):

$$
\left\{\begin{array}{l}
z^{\prime}(t)+k_{1} z(t)-k_{2} z(t-r)=\alpha f(z(t))+\beta g(z(t-r)), \\
z(s)=z_{0}(s), s \in[-r, 0],
\end{array}\right.
$$

where $k_{1}, k_{2}, \alpha$, and $\beta$ are some arbitrarily given constants, and $f(z)$ and $g(z)$ satisfy

$$
|f(z)| \leq C|z|^{m} \text { and }|g(z)| \leq C|z|^{n}, \quad m>1, n>1 .
$$

We then obtain nonlinear stability as follows.

Lemma 3.9. Let $z(t)$ be the solution of (3.32). If $\left|k_{2}\right| \leq k_{1}$ with any time-delay $r>0$, or if $\left|k_{2}\right|>k_{1}$ but with small time-delay $0<r<\bar{r}$, where $\bar{r}$ is defined in (3.30), then it holds that

$$
|z(t)| \leq C\left\|z_{0}\right\|_{L^{\infty}(-r, 0)} e^{-\nu t}, \quad t>0,
$$

for some constant $0<\nu=\nu\left(k_{1}, k_{2}, r\right)<k_{1}$, provided $\left\|z_{0}\right\|_{L^{\infty}} \ll 1$.

Proof. Inequality (3.34) can be proved by the iteration technique with the energy estimates based on Lemma 3.8 and the Banach fixed point theorem. It can be also proved by the $C^{1}$-perturbed Grownwall inequality; see the textbook [13]. So, the details are omitted.

Now we are at the stage to derive the time-exponential decay of $u(t, \xi)$ for $\xi=\infty$.

Lemma 3.10. If $d \geq\left|b^{\prime}\left(v_{+}\right)\right|$with arbitrary time-delay $r>0$, or if $d<\left|b^{\prime}\left(v_{+}\right)\right|$ but with small time-delay $0<r<\bar{r}$, where $\bar{r}$ is defined in (1.14), then

$$
|u(t, \infty)| \leq C M_{u}(0) e^{-\mu_{2} t}, t>0,
$$

for some $0<\mu_{2}=\mu_{2}\left(p, d, r, b^{\prime}\left(v_{+}\right)\right)<d$, provided $M_{u}(0) \ll 1$.

Proof. For (3.21), in order to apply the nonlinear Halanay inequality (Lemma 3.9), we take $k_{1}=d$ and $k_{2}=b^{\prime}\left(v_{+}\right)<0$ in (3.32). Thanks to Lemma 3.9, either when $d \geq\left|b^{\prime}\left(v_{+}\right)\right|$with any time-delay $r>0$, or when $d<\left|b^{\prime}\left(v_{+}\right)\right|$with small time-delay $0<r<\bar{r}$, then

$$
|u(t, \infty)|=|z(t)| \leq C M_{u}(0) e^{-\mu_{2} t}, t>0,
$$


for some constant $0<\mu_{2}=\mu_{2}\left(p, d, r, b^{\prime}\left(v_{+}\right)\right)<d$, provided $M_{u}(0) \ll 1$. The proof is complete.

Since

$$
\lim _{\xi \rightarrow \infty}\left|e^{\mu_{2} t} u(t, \xi)-e^{\mu_{2} t} u(t, \infty)\right|=0 \text { uniformly in } t \in[0, T],
$$

namely, for any given positive number $\varepsilon>0$, there exists a number $x_{0}=x_{0}(\varepsilon) \gg 1$ but independent of $t$, such that when $\xi \geq x_{0}$,

$$
\left|e^{\mu_{2} t} u(t, \xi)-e^{\mu_{2} t} u(t, \infty)\right|<\varepsilon,
$$

which implies

$$
\left|e^{\mu_{2} t}\right| u(t, \xi)\left|-e^{\mu_{2} t}\right| u(t, \infty)|| \leq\left|e^{\mu_{2} t} u(t, \xi)-e^{\mu_{2} t} u(t, \infty)\right|<\varepsilon .
$$

Notice that, from (3.35), $e^{\mu_{2} t}|u(t, \infty)| \leq C M_{u}(0)$ is uniformly bounded with respect to $t$; we then immediately obtain

$$
e^{\mu_{2} t}|u(t, \xi)| \leq C M_{u}(0)+\varepsilon \text { for } \xi \geq x_{0}, t \in[0, T] .
$$

Now, let us take $\varepsilon=M_{u}(0)$; we immediately prove the following lemma.

LEMma 3.11. If $d \geq\left|b^{\prime}\left(v_{+}\right)\right|$with arbitrary time-delay $r>0$, or if $d<\left|b^{\prime}\left(v_{+}\right)\right|$ but with small time-delay $0<r<\bar{r}$, where $\bar{r}$ is defined in (1.14), then there exists large number $x_{0} \gg 1$ (independent of $t$ ) such that

$$
\sup _{\xi \in\left[x_{0}, \infty\right)}|u(t, \xi)| \leq C M_{u}(0) e^{-\mu_{2} t}, t>0 .
$$

Proof of Proposition 2.3. Combining (3.37), (3.18), and (3.20), we immediately prove (2.12), namely,

$$
\begin{aligned}
& \|u(t)\|_{C}^{2}+\|u(t)\|_{H_{w}^{2}}^{2}+\int_{0}^{t} e^{-2 \mu(t-s)}\|u(s)\|_{H_{w}^{2}}^{2} d s \\
& \leq C_{0} e^{-2 \mu t}\left(\max _{s \in[-r, 0]}\left\|u_{0}(s)\right\|_{C}^{2}+\left\|u_{0}(0)\right\|_{H_{w}^{2}}^{2}+\int_{-r}^{0}\left\|u_{0}(s)\right\|_{H_{w}^{2}}^{2} d s\right)
\end{aligned}
$$

for some positive constant $C_{0}$, where $\mu$ is taken as $0<\mu \leq \min \left\{\mu_{1}, \mu_{2}\right\}$. The proof of Proposition 2.3 is complete.

4. Proof of uniqueness of traveling waves. In this section, we prove Corollary 1.3. As a special case, Corollary 1.5 directly follows from Corollary 1.3.

Let $\phi_{1}(x+c t)$ and $\phi_{2}(x+c t)$ be two different traveling waves with the same speed $c>c_{*}$ and the same exponential decay at $-\infty$ :

$$
\phi_{1}(\xi)=A e^{-\lambda_{1}|\xi|} \text { as } \xi \rightarrow-\infty
$$

and

$$
\phi_{2}(\xi)=B e^{-\lambda_{1}|\xi|} \text { as } \xi \rightarrow-\infty
$$

for some positive constants $A$ and $B$, where $\lambda_{1}=\lambda_{1}(c)>0$ is defined in (1.10). Let us shift $\phi_{2}(x+c t)$ to $\phi_{2}\left(x+c t+x_{2}\right)$ with some constant shift $x_{2}$. By taking $\xi \rightarrow-\infty$, obviously $\xi+x_{2}<0$. Then

$$
\phi_{2}\left(\xi+x_{2}\right)=B e^{-\lambda_{1}\left|\xi+x_{2}\right|}=B e^{\lambda_{1}\left(\xi+x_{2}\right)}=B e^{\lambda_{1} x_{2}} e^{-\lambda_{1}|\xi|}=A e^{-\lambda_{1}|\xi|} \quad \text { as } \xi \rightarrow-\infty
$$


by selecting $x_{2}$ as

$$
x_{2}=\frac{1}{\lambda_{1}} \ln \frac{A}{B} .
$$

Thus, we have

$$
\left|\phi_{2}\left(\xi+x_{2}\right)-\phi_{1}(\xi)\right|=O(1) e^{-\alpha|\xi|} \text { for } \alpha>\lambda_{1} \text { as } \xi \rightarrow-\infty .
$$

This implies

$$
\phi_{2}\left(\xi+x_{2}\right)-\phi_{1}(\xi) \in C(R) \cap H_{w}^{1}(R) .
$$

Now we take the initial data for (1.1) by

$$
v_{0}(s, x)=\phi_{2}\left(x+c s+x_{0}\right), x \in R, s \in[-r, 0] .
$$

Obviously, with such selected initial data, the corresponding solution to (1.1) is

$$
v(t, x)=\phi_{2}\left(x+c t+x_{2}\right) .
$$

Applying the stability theorem, Theorem 1.2 , when $d \geq\left|b^{\prime}\left(v_{+}\right)\right|$with any time-delay $r>0$, or when $d<\left|b^{\prime}\left(v_{+}\right)\right|$but with $0<r<\bar{r}$, then

$$
\lim _{t \rightarrow \infty} \sup _{x \in R}\left|\phi_{2}\left(x+c t+x_{2}\right)-\phi_{1}(x+c t)\right|=0,
$$

namely, $\phi_{2}\left(x+c t+x_{2}\right)=\phi_{1}(x+c t)$ for all $x \in R$ as $t \gg 1$. This proves the uniqueness of the traveling waves up to a constant shift.

5. Numerical computations. In this section, we carry out some numerical simulations in different cases. We take $b(v)=p v e^{-a v}$. Without loss of generality, we may always fix $d=1, D=1, a=1$, but leave $p, r$, and the initial data $v_{0}(s, x)$ to be selectable. The initial data are taken as

$$
v_{0}(s, x)=\frac{v_{+}}{1+e^{-k x}}+0.1(\sin x) e^{-0.001(x-500)^{2}}, \quad s \in[-r, 0],
$$

which implies

$$
\left|v_{0}(s, x)-v_{ \pm}\right|=O(1) e^{-k|x|} \text { as } x \rightarrow \pm \infty,
$$

where $k>0$ can be selected in different cases. According to our stability theorem, Theorem 1.4, when $e<\frac{p}{d} \leq e^{2}$, for any time-delay $r>0$, the solution $v(t, x)$ is expected to converge time-asymptotically to a monotone/nonmonotone traveling wave with $c>c_{*}$, where the wave is monotone for small time-delay $r<\underline{r}$, but possibly nonmonotone (oscillating around $v_{+}$) if $r \geq \underline{r}$, where $\underline{r}>0$ is given in (1.7), i.e.,

$$
d\left(\ln \frac{p}{d}-1\right) \underline{r} e^{\underline{r} d+1}=1 .
$$

Inspired by Gomez and Trofimchuk's analysis [7], there exists a certain region $\mathcal{D}$ (see also the summary presented in Theorem 1.1) such that the wavefronts $\phi(x+c t)$ are monotone if and only if the speed $c$ and the time-delay $r$ belong to $\mathcal{D}$; namely, even though $r>\underline{r}$, if $c \in\left[c_{*}, c^{*}\right]$, where $c_{*}$ and $c^{*}$ are defined in (1.9) and (1.12), the wavefronts are still monotone, but the wavefronts are oscillatory around $v_{+}$if the 
TABLE 1

Different cases for selection of $p, r$, and the initial data $v_{0}(s, x)$.

\begin{tabular}{|c|c|c|c|c|c|l|}
\hline Case & $p$ & $r$ & Zone of $\frac{p}{d}$ & Zone of $r$ & $k$ for $v_{0}$ & Behavior of $v(t, x)$ \\
\hline 1 & 6 & 0.5 & $e<\frac{p}{d} \leq e^{2}$ & $\underline{r}<r$ & $k=1$ & $\begin{array}{l}\text { monotone wave with } \\
c \approx 2.1 \in\left[c_{*}, c^{*}\right]\end{array}$ \\
\hline 2 & 6 & 0.5 & $e<\frac{p}{d} \leq e^{2}$ & $\underline{r}<r$ & $k=0.075$ & $\begin{array}{l}\text { oscillatory wave with } \\
c \approx 22 \gg c^{*}\end{array}$ \\
\hline 3 & 6 & 10 & $e<\frac{p}{d} \leq e^{2}$ & $\underline{r} \ll r$ & $k=1$ & $\begin{array}{l}\text { oscillatory wave with } \\
c \approx 0.35\end{array}$ \\
\hline 4 & 10 & 0.2 & $\frac{p}{d}>e^{2}$ & $r<\underline{r}$ & $k=1$ & $\begin{array}{l}\text { monotone wave with } \\
c \approx 3.1\end{array}$ \\
\hline 5 & 10 & 0.5 & $\frac{p}{d}>e^{2}$ & $\underline{r}<r<\bar{r}$ & $k=1$ & $\begin{array}{l}\text { oscillatory wave with } \\
c \approx 2.1\end{array}$ \\
\hline 6 & 10 & 3 & $\frac{p}{d}>e^{2}$ & $r>\bar{r}$ & $k=1$ & $\begin{array}{l}\text { unknown case in Theo- } \\
\text { rem 1.4 }\end{array}$ \\
\hline 7 & 10 & 10 & $\frac{p}{d}>e^{2}$ & $r \gg \bar{r}$ & $k=1$ & $\begin{array}{l}\text { unknown case in Theo- } \\
\text { rem 1.4 }\end{array}$ \\
\hline
\end{tabular}

wave speed $c$ is big, $c>c^{*}$, or the time-delay is big, $r \gg 1$, such that $(c, r) \notin \mathcal{D}$. Here, we will numerically test it in three cases (see Cases 1-3 below). On the other hand, when $\frac{p}{d}>e^{2}$ and $0<r<\bar{r}$, where

$$
\bar{r}:=\left(\pi-\arctan \sqrt{\ln \frac{p}{d}\left(\ln \frac{p}{d}-2\right)}\right) /\left(d \sqrt{\ln \frac{p}{d}\left(\ln \frac{p}{d}-2\right)}\right),
$$

our stability theorem, Theorem 1.4, predicates that the monotone/nonmonotone traveling waves are still exponentially stable. In this case, we will carry out two numerical experiments to confirm it. One (Case 4) shows that, with $0<r<\underline{r}$, the solution $v(t, x)$ converges to a monotone traveling wave, but the other (Case 5) shows the convergence to an oscillatory traveling wave if $\underline{r}<r<\bar{r}$. Finally, in the last two cases (Cases 6 and 7), when $\frac{p}{d}>e^{2}$ with $r>\bar{r}$, we numerically show that the solution will always chaotically oscillate and never behave like an oscillatory traveling wave. We observe that when the time-delay $r$ is getting bigger, then the amplitudes and the widths of those chaotic oscillations become larger. Unfortunately we are not clear about the asymptotic profile of the solution when $\frac{p}{d}>e^{2}$ and $r>\bar{r}$. The study on this case is quite difficult and remains an open problem. Now we summarize all abovementioned cases in Table 1 and discuss each case individually with many numerical simulations.

The adopted numerical scheme is the unconditionally stable Crank-Nicolson method to a finite computational domain $[-L, L]$. Here, we take $L$ to be at least 800 so that the computational domain is sufficiently large to avoid a numerical boundary effect. The spatial step size is chosen to be $\Delta x=0.1$, and the temporal step size is $\Delta t=0.05$.

Case 1: $e<\frac{p}{d} \leq e^{2}$ with time-delay $r>\underline{r}$ and $c \in\left[c_{*}, c^{*}\right]$; the case of convergence to a monotone traveling wave. In this case, we take $p=6, r=0.5$, and $k=1$ in the initial data $v_{0}(s, x)$ (see (5.1)), which may allow us to expect the solution to behave like a traveling wave with small wave speed, because, in our experience, when the initial perturbation decays faster at $x=-\infty$, the solution usually 
(a)

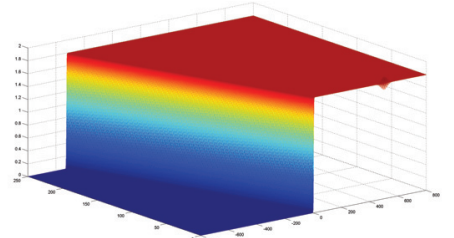

(a)

FIG. 2. Case 1: $e<\frac{p}{d}=6<e^{2}$ with small time-delay $r=0.5>\underline{r}=0.33304 \cdots$ and faster decaying initial data $v_{0}(s, x)$. The solution $v(t, x)$ behaves like a stable monotone traveling wave $\phi(x+c t)$ with small wave speed $c \in\left[c_{*}, c^{*}\right]$. (a) Three-dimensional mesh of $v(t, x)$, and (b) the contour of $v(t, x)$.

(a)

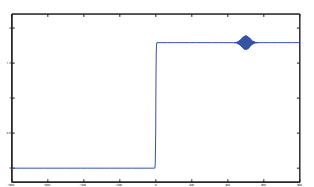

(d)

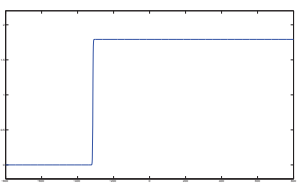

(b)

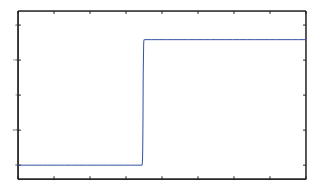

(e)

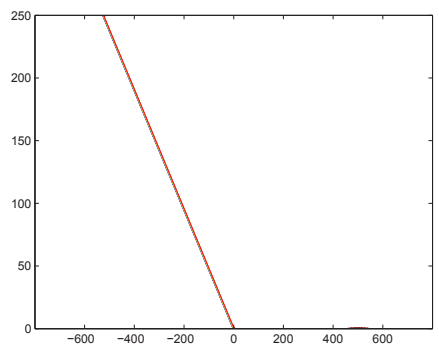

(b)

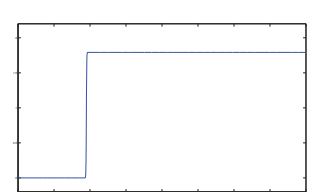

(f)

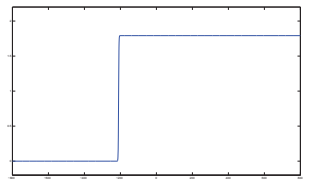

(c)

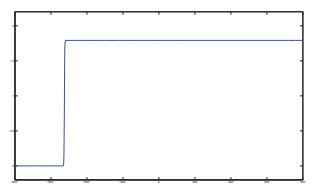

FIG. 3. Case 1: $e<\frac{p}{d}=6<e^{2}$ with small time-delay $r=0.5>\underline{r}=0.33304 \cdots$. From (a) to (f), the solution $v(t, x)$ plotted at times $t=0,50,100,150,200,250$, which behaves like a stable monotone traveling wave (no change of the wave's shape for the sense of stability) and travels from right to left.

travels slower. We compute the solution $v(t, x)$ up to time $t=250$. The numerical simulations presented in Figures 2 and 3 confirm that the solution of Nicholson's blowflies equations (1.1) and (1.2) converges to a monotone traveling wave.

Figure 2 (a) shows that, after a small initial oscillation, the solution $v(t, x)$ quickly behaves like a monotone traveling wave which travels from right to left (i.e., the wave speed $c>0$ ). From the contour map shown in Figure 2 (b), we observe that the interface region of left and right states, $v_{-}$and $v_{+}$, travels from right to left. The contour lines are straight and the width of interface region at each time appears constant. Both facts indicate that the solution's profile remains unchanged for larger times. The wave speed can be also estimated from the contour line as $c \approx 2.1$. Again, in Figure 2 (a), and particularly in Figure 3, the increasing shape of the solution $v(t, x)$, at different times $t=50,100,150,200$, and 250, is the same and travels from right to left. These phenomena indicate that the solution $v(t, x)$ of Nicholson's blowflies equations (1.1) and (1.2) behaves like a certain monotone traveling wave $\phi(x+c t)$ with $c \approx 2.1$; in other words, the solution $v(t, x)$ converges time-asymptotically to the monotone traveling wave $\phi(x+c t)$ with $c \approx 2.1$. This also completely matches what was predicated in [7] for the case of existence of monotone traveling waves. In fact, it can be verified that $\frac{p}{d}$ satisfies $e<\nu_{0}=2.808 \cdots<\frac{p}{d}=6<e^{2}$ and $r=0.5>\underline{r}=0.33304 \cdots$ and the critical wave speed (see (1.9)) $c_{*}=1.8 \cdots$, and 
$c^{*}=2.4 \cdots($ see $(1.12))$, and then $c \approx 2.1 \in\left[c_{*}, c^{*}\right]=[1.8 \cdots, 2.4 \cdots]$, the region to possess the monotone traveling waves.

Case 2: $e<\frac{p}{d} \leq e^{2}$ with $r>\underline{r}$ and $c>c^{*}$; the case of convergence to an oscillatory traveling wave. We take $p=6$ and $r=0.5$ to be the same as in Case 1 , but $k=0.075$ in the initial data $v_{0}(s, x)$ (see (5.1)), which may allow us to expect the solution to behave as a traveling wave with a large wave speed, because the initial data decays to 0 slowly like $e^{-0.075|x|}$. We compute the solution $v(t, x)$ for $x$ from -5000 to 5000 and for $t$ from 0 to 400 . Figures 4 and 5 both indicate that the solution $v(t, x)$ behaves like an oscillatory traveling wave with small amplitude of oscillations. In order to easily see those oscillations, we enlarge the part of oscillations for the solution in Figure 5 (b). Notice that the shape of the solution at different large times stays the same, and the oscillations never disappear. This means that the solution $v(t, x)$ converges to its corresponding oscillatory traveling wave $\phi(x+c t)$. From Figure 4 (b), the contour line estimates the wave speed to be really big like $c \approx 22$. This also completely matches the theoretical prediction in [7], because it is just the case to possess the nonmonotone traveling waves for $e<\nu_{0}=2.808 \cdots<\frac{p}{d}=6<e^{2}$, $r=0.5>\underline{r}=0.33304 \cdots$, and $c \approx 22 \gg c^{*}=2.4 \cdots$.

Case 3: $e<\frac{p}{d} \leq e^{2}$ with large time-delay $r \gg \underline{r}$; the case of convergence to an oscillatory traveling wave. In this case, we still take $p=6$, but $r=10$. The initial data is the same as in Case 1 . So, we verify that $e<\nu_{0}=$ $2.808 \cdots<\frac{p}{d}=6<e^{2}$ and $r=10 \gg \underline{r}=0.33304 \cdots$. The solution is expected to converge to a certain oscillatory traveling wave. We compute it up to time $t=500$.

The numerical simulations in Figures 6 and 7 present the large time behavior of the solution $v(t, x)$ of Nicholson's blowflies equations (1.1) and (1.2). It can be easily observed from Figure 6 (a) and Figure 7 that, after the initial oscillations, the solution $v(t, x)$ develops gradually with some small oscillations at the interface region of right state $v_{+}$, but these oscillations never disappear and keep the same shape. At times not shown in Figure 7, the shape of solution $v(t, x)$ looks identical, but travels from right to left.

In Figure 7, since the oscillations of the solution $v(t, x)$ around the interface region of $v_{+}$are slow and the amplitudes of oscillation are small, it is hard for us to see these oscillatory phenomena. In Figure 8(a) we enlarge these oscillation parts of the solution $v(t, x)$ for $t=300,350,400,450$, and 500 . It is also easily seen that the shape of these oscillations for $v(t, x)$ at different times is the same. Furthermore, we test the maximum of oscillations for $v(t, x)$ :

$$
h(t)=\max _{x \in[-L, L]} v(t, x)-v_{+}
$$

As shown in Figure 8 (b), we can observe that the maximum amplitude of oscillations above $v_{+}$(i.e., the function of $h(t)$ ) looks like a positive constant after a large time. This means that the oscillations never disappear, and keep the same maximum amplitude. From the contour line shown in Figure 6 (b), we can estimate the traveling speed for the solution $v(t, x)$ as $c \approx 0.35$. By solving $c_{*}(r)=c^{*}(r)$, we get the unique intersection point $r_{0}=0.63178 \cdots$. Since $r=10 \gg r_{0}=0.63178 \cdots$ is out of the domain $\mathcal{D}=\left[0, r_{0}\right] \times\left[c_{*}, c^{*}\right]$ for the existence of monotone traveling waves (see Figure 1 ), the wavefront is expected to be oscillatory around $v_{+}$[7]. In fact, our numerical results show that the original solution $v(t, x)$ behaves like such a stable oscillatory traveling wave $\phi(x+c t)$ with $c \approx 0.35$ after a long time, which also perfectly match 
(a)

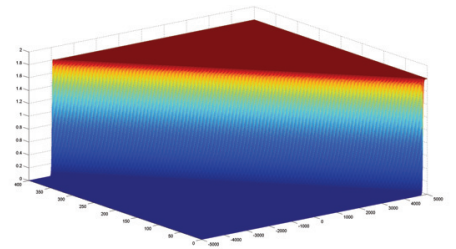

FIG. 4. Case 2: $e<\frac{p}{d}=6<e^{2}$ with small time-delay $r=0.5>\underline{r}=0.33304 \cdots$ and slowly decaying initial data $v_{0}(s, x)$. The solution $v(t, x)$ behaves like a stable oscillatory traveling wave $\phi(x+c t)$ with large speed $c>c^{*}$. (a) Three-dimensional mesh of $v(t, x)$, and (b) the contour of $v(t, x)$.

(a)

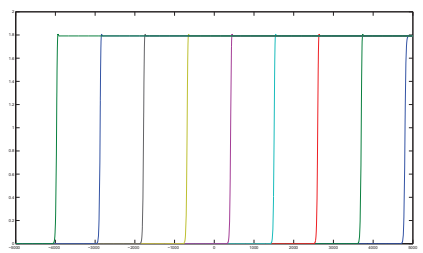

(b)

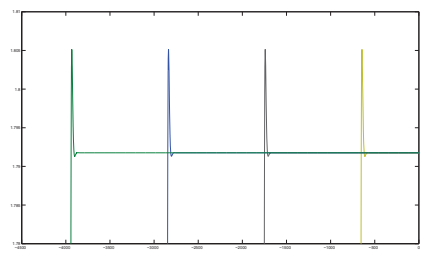

FIG. 5. Case 2: $e<\frac{p}{d}=6<e^{2}$ with small time-delay $r=0.5>\underline{r}=0.33304 \cdots$ and slowly decaying initial data $v_{0}(s, x)$. (a) Two-dimensional solution $v(t, x)$ plotted at times $t=$ $0,50,100,150,200,250,300,350,400$, which behaves like a stable oscillatory traveling wave (no change of the wave's shape for the sense of stability) and travels from right to left. (b) Enlargement of oscillation parts of $v(t, x)$ around $v_{+}$for time $t$ at $100,200,300,400$.

(a)

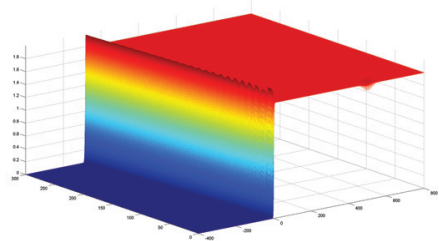

(b)

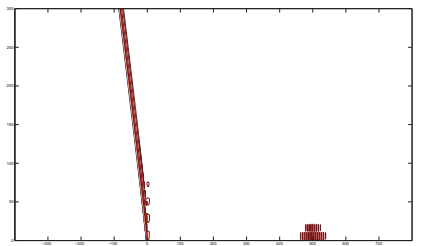

FIG. 6. Case 3: $e<\frac{p}{d}=6<e^{2}$ with large time-delay $r=10 \gg \underline{r}=0.33304 \cdots$. The solution $v(t, x)$ behaves like stable oscillatory traveling wave $\phi(x+c t)$. (a) Three-dimensional mesh of $v(t, x)$, and (b) the contour of $v(t, x)$.

(a)

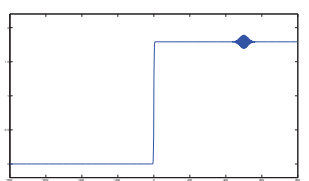

(d)

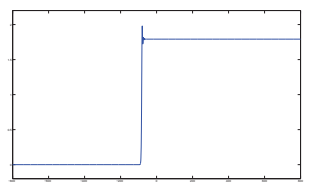

(b)

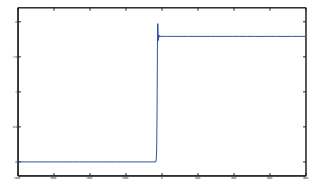

(e)

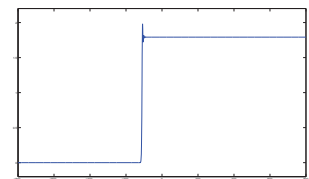

(c)

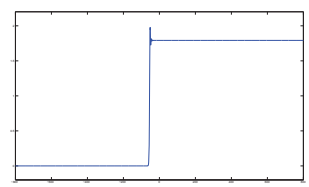

(f)

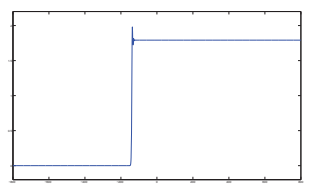

FIG. 7. Case 3: $e<\frac{p}{d}=6<e^{2}$ with large time-delay $r=10 \gg \underline{r}=0.33304 \cdots$. From (a) to (f), the solution $v(t, x)$ plotted at times $t=0,300,235,400,450,500$, which behaves like a stable oscillatory traveling wave (no change of the wave's shape for the sense of stability) and travels from right to left. 
(a)

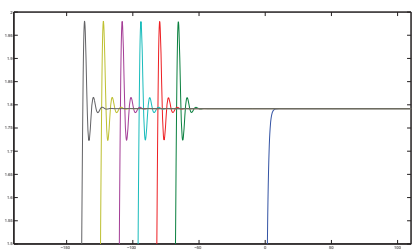

(b)

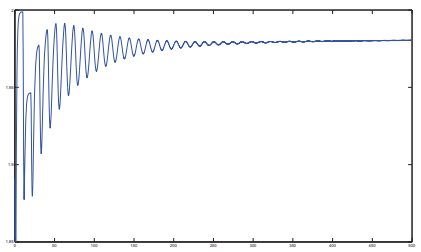

FIG. 8. Case 3: $e<\frac{p}{d}=6<e^{2}$ with large time-delay $r=10 \gg \underline{r}=0.33304 \cdots$. (a) Enlargement of oscillations presented in Figure 7 for the solution $v(t, x)$ plotted at times $t=0,300$, $350,400,450$, and 500 (from right to left), and (b) the graph of $h(t)$ given in (5.2).

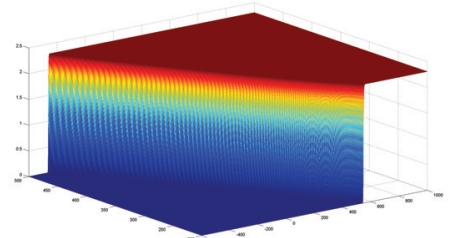

(a)

FIG. 9. Case 4: $\frac{p}{d}=10>e^{2}$ with small time-delay $r=0.2<\underline{r}=0.22546 \cdots$. (a) Threedimensional mesh of $v(t, x)$ for $t$ from 200 to 500, and $x$ from -600 to 1000 , and (b) the contour of $v(t, x)$.

what Gomez and Trofimchuk [7] theoretically analyzed, and demonstrate the stability of oscillatory traveling wave $\phi(x+c t)$ with $c \approx 0.35$.

Case 4: $\frac{p}{d}>e^{2}$ with small time-delay $r<\underline{r}$; the case of convergence to a monotone traveling wave. Here, we take $p=10, r=0.2$, and $v_{0}(s, x)$ the same as in Case 1 . We can verify that $\frac{p}{d}=10>e^{2}$ and $r=0.2<\underline{r}=0.22546 \cdots$. Thus, the solution is expected to behave like a certain monotone traveling wave. In fact, the numerical simulations shown in Figures 9 and 10 perfectly confirm this theoretical prediction, and the contour line in Figure $9(\mathrm{~b})$ estimates the wave speed to be $c \approx 3.1$.

Case 5: $\frac{p}{d}>e^{2}$ with $\underline{r}<r<\bar{r}$; the case of convergence to an oscillatory traveling wave. In this case, the parameters are taken as $p=10, r=0.5$, and $v_{0}(s, x)$ as in Case 1 . Thus, $\frac{p}{d}=10>e^{2}$, and $r$ satisfies

$$
0.22546 \cdots=\underline{r}<r=0.5<\bar{r}=2.93048 \cdots
$$

Figures 11 and 12 show that the solution $v(t, x)$ behaves numerically like a stable nonmonotone wave traveling from right to left. Since the amplitude of oscillation around $v_{+}$is very small, we enlarge it in Figure 12 (b). Notice that when $t=$ $750,900,1050,1200,1350$, we find that the amplitude of oscillations for the solution $v(t, x)$ around $v_{+}$for the parts $v \geq v_{+}$is really small,

$$
h(t):=\max _{x \in[-L, L]} v(t, x)-v_{+} \approx 0.006
$$

but almost identical for each time $t$, and never disappears. This indicates that, after a long time, the solution $v(t, x)$ stably behaves like an oscillatory traveling wave with small amplitude of oscillations. 

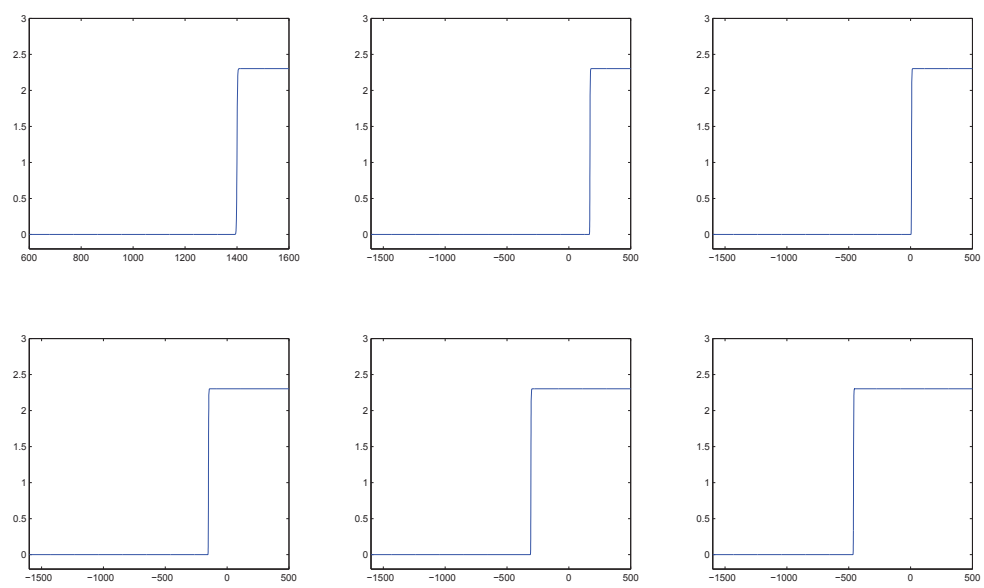

Fig. 10. Case 4: $\frac{p}{d}=10>e^{2}$ with small time-delay $r=0.2<\underline{r}=0.22546 \cdots$. Twodimensional solution $v(t, x)$ plotted at times $t=0,300,350,400,450,460$. The solution behaves like a stable monotone wavefront traveling from right to left.

Case 6: $\frac{p}{d}>e^{2}$ with a big time-delay $r=3>\bar{r}$; the case of chaotic oscillations. Let $p=10$ and $r=3$, and let the initial data $v_{0}(s, x)$ be the same as in Case 1, such that

$$
\frac{p}{d}>e^{2}, \quad r=3>\bar{r}=2.93048 \cdots .
$$

However, as shown in Figures 13, 14, and 15, the behavior of solution $v(t, x)$ is oscillatory but chaotic. Particularly, from Figures 14 and 15, we see that there is much disorder oscillation around $x=200$, and the intervals of oscillations around the location $x=200$ expand gradually. Such chaotic oscillations tell us that the solution doesn't behave like an oscillatory traveling wave.

Case 7: $\frac{p}{d}>e^{2}$ with time-delay $r=10>\bar{r}$; the case of chaotic oscillation. This case basically is the same as Case 6 by taking $p=10, v_{0}(s, x)$ the same as in Case 6, but $r=10>\bar{r}$ (a bit big), such that

$$
\frac{p}{d}>e^{2}, \quad r=10>\bar{r}=2.93048 \cdots .
$$

From Figures 16, 17, and 18, we observe that the behavior of solution $v(t, x)$ is very oscillatory and chaotic. Comparing Cases 7 and 6, we know that the larger the timedelay, the bigger the oscillation amplitudes, and the wider the oscillation intervals in $x$. Obviously, the solution doesn't behave like an oscillatory traveling wave, and its asymptotic profile is still a mystery.

Acknowledgments. The authors would like to express their sincere thanks to two anonymous referees for their valuable and helpful comments, which led to a major and important improvement of the paper. This work was initiated when MM visited the Hong Kong Polytechnic University and National Chiao Tung University in the fall of 2012 for his sabbatical leave. He would like to thank them for their great hospitality. 
(a)

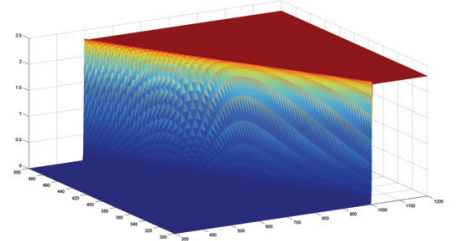

(b)

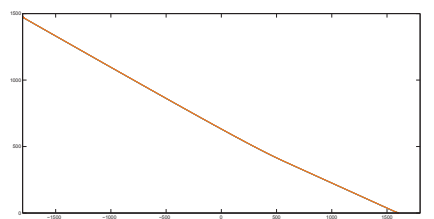

FIG. 11. Case 5: $\frac{p}{d}=10>e^{2}$ with small time-delay $\underline{r}<r=0.5<\bar{r}$. (a) Three-dimensional mesh of $v(t, x)$ for $t$ from 0 to 1500 and $x$ from -1800 to 1800 , and (b) the contour of $v(t, x)$.

(a)

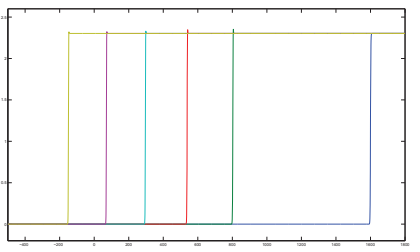

(b)

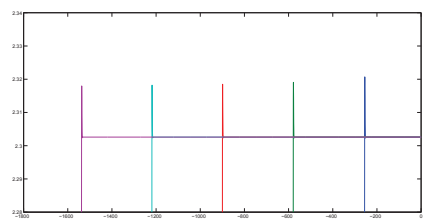

FIG. 12. Case 5: $\frac{p}{d}=10>e^{2}$ with small time-delay $\underline{r}<r=0.5<\bar{r}$. (a) Two-dimensional solution $v(t, x)$ plotted at times $t=0,300,400,500,600,700$. The solution behaves like a stable nonmonotone wavefront traveling from right to left, but the oscillation amplitudes around $v_{+}$are small. (b) Enlargement of the oscillation parts of the solution $v(t, x)$ plotted at times $t=$ $750,900,1050,1200,1350$. Although each amplitude of oscillation at different times for the solution is really small, they are almost equivalent.

(a)

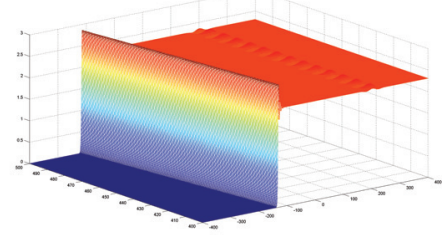

(b)

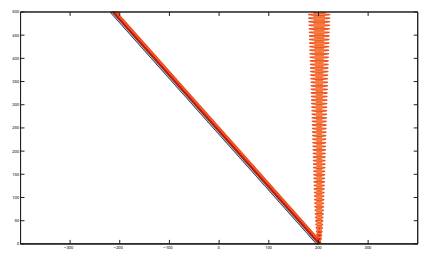

FIG. 13. Case 6: $\frac{p}{d}=10>e^{2}$ with big time-delay $r=3>\bar{r}$. (a). Three-dimensional mesh of $v(t, x)$ for $t$ from 400 to 500 and $x$ from -400 to 400 , and (b) the contour of $v(t, x)$.

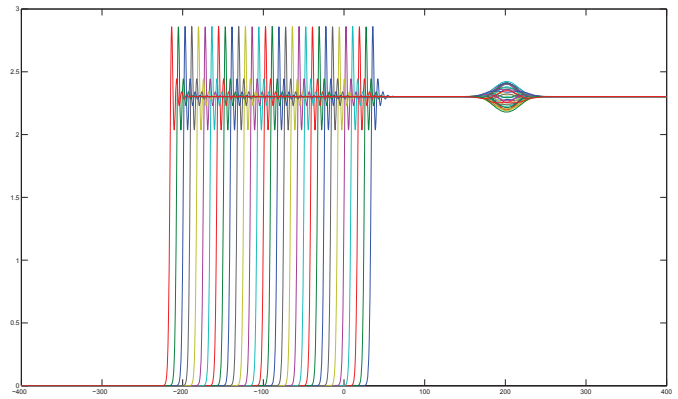

FIG. 14. Case 6: $\frac{p}{d}=10>e^{2}$ with big time-delay $r=3>\bar{r}$. Two-dimensional solution $v(t, x)$ plotted at times $t=200,210,220, \ldots, 500$ (the step is 10). The solution behaves like some chaotic oscillations. 

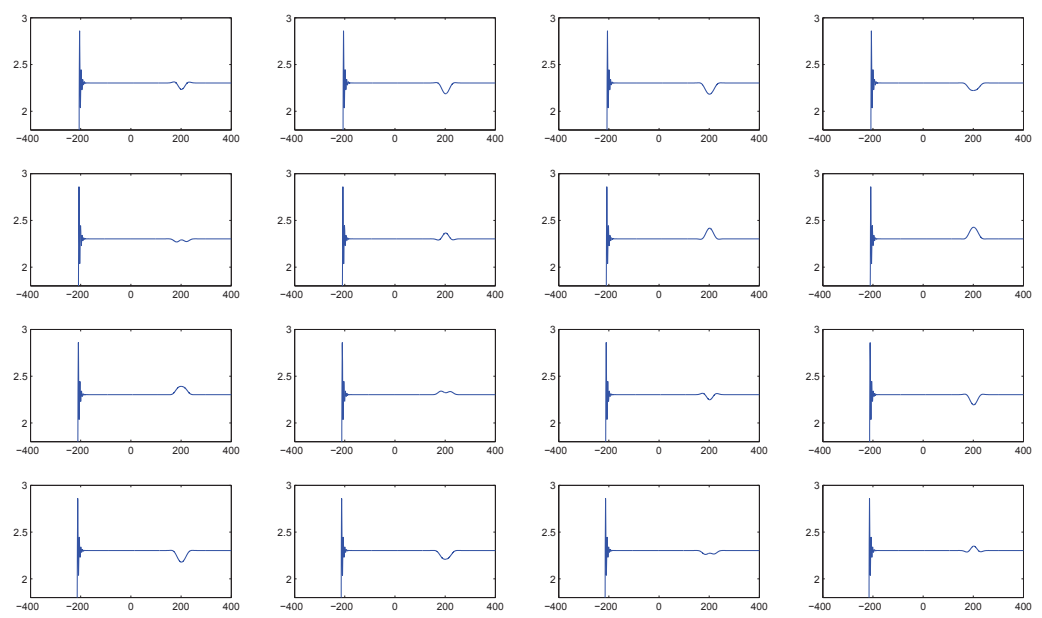

FIG. 15. Case 6: $\frac{p}{d}=10>e^{2}$ with big time-delay $r=3$. Two-dimensional solution $v(t, x)$ plotted at times $t=288.75,289.5,2290.25, \ldots, 300$ (the step is 0.75 ). The solution behaves like some chaotic oscillations.

(a)

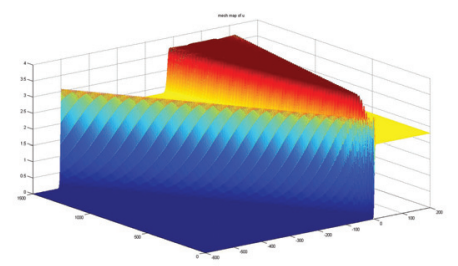

(b)

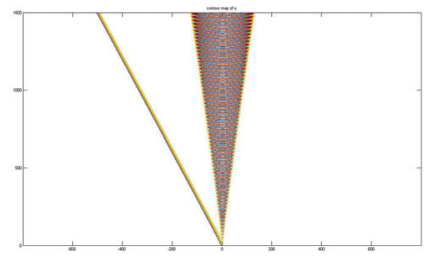

(a) Three-dimensional mesh of $(t, x)$, and (b) the contour of $v(t, x)$.

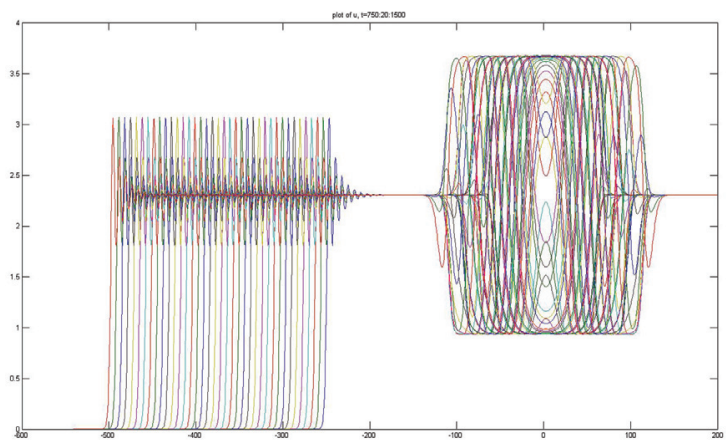

FIG. 17. Case 7: $\frac{p}{d}=10>e^{2}$ with big time-delay $r=10>\bar{r}$. Two-dimensional solution $v(t, x)$ plotted at times $t=750,770,790, \ldots, 1500$ with identical step of 20 . The solution behaves like some chaotic oscillations. 

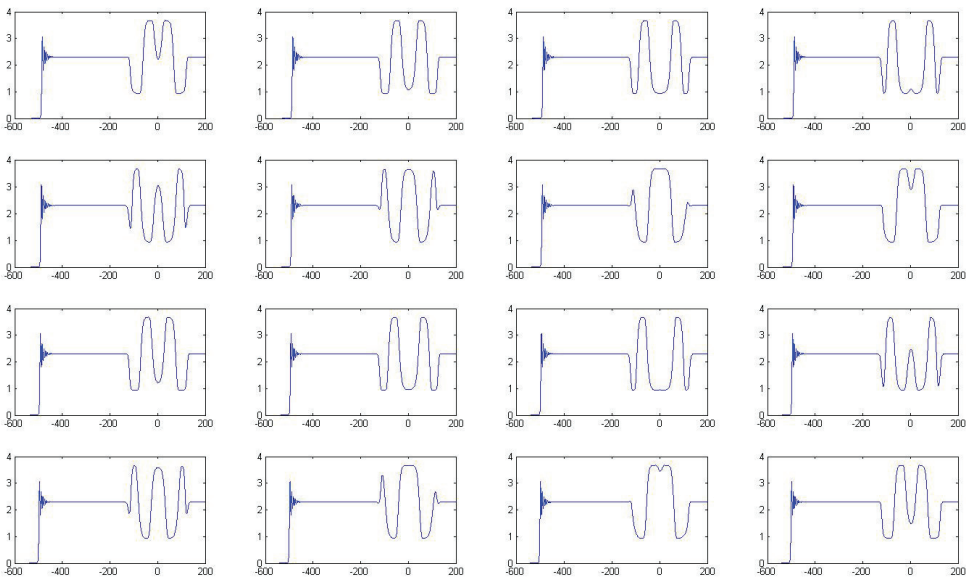

FIG. 18. Case 7: $\frac{p}{d}=10>e^{2}$ with big time-delay $r=10>\bar{r}$. Two-dimensional solution $v(t, x)$ plotted at times $t=1445,1448,1451, \ldots, 1560$ with identical step of 3 . The solution behaves like some chaotic oscillations.

\section{REFERENCES}

[1] M. Aguerrea, C. Gomez, and S. Trofimchuk, On uniqueness of semi-wavefronts, Math. Ann., 354 (2012), pp. 73-109.

[2] F. G. Boese, Some stability charts and stability conditions for a class of difference-differential equations, ZAMM Z. Angew. Math. Mech., 67 (1987), pp. 56-59.

[3] I-L. Chern And M. MeI, Asymptotic stability of critical viscous shock waves for a degenerate hyperbolic viscous conservation laws, Comm. Partial Differential Equations, 23 (1998), pp. 869-886.

[4] T. FARIA, W. HuAng, AND J. Wu, Traveling waves for delayed reaction-diffusion equations with global response, Proc. Roy. Soc. London Ser. A, 462 (2006), pp. 229-261.

[5] T. FARIA AND S. Trofimchuk, Nonmonotone traveling waves in single species reactiondiffusion equation with delay, J. Differential Equations, 228 (2006), pp. 357-376.

[6] T. Faria and S. Trofimchuk, Positive heteroclinics and traveling waves for scalar population models with a single delay, Appl. Math. Comput., 185 (2007), pp. 594-603.

[7] A. Gomez and S. Trofimchuk, Global Continuation of Monotone Wavefronts, preprint, arXiv:1210.6419v1, 2012.

[8] K. Gopalsamy, Stability and Oscillation in Delay Differential Equations of Population Dynamics, Kluwer Academic, Dordrecht, 1992.

[9] S. A. Gourley, Linear stability of traveling fronts in an age-structured reaction-diffusion population model, Quart. J. Mech. Appl. Math., 58 (2005), pp. 257-268.

[10] S. A. Gourley, J. W.-H. So, AND J. Wu, Nonlocality of reaction-diffusion equations induced by delay: Biological modeling and nonlinear dynamics, in Contemporary Mathematics, Thematic Surveys, D. V. Anosov and A. Skubachevskii, eds., Kluwer Plenum, 2003, pp. 84120 (in Russian); J. Math. Sci., 124 (2004), pp. 5119-5153 (in English).

[11] S. A. Gourley and J. Wu, Delayed nonlocal diffusive system in biological invasion and disease spread, Fields Inst. Commun., 48 (2006), pp. 137-200.

[12] W. S. C. Gurney, S. P. Blythe, And R. M. Nisbet, Nicholson's blowflies revisited, Nature, 287 (1980), pp. 17-21.

[13] J. K. Hale and S. M. Verduyn Lunel, Introduction to Functional Differential Equations, Springer, New York, 1993.

[14] F.-M. Huang, M. MeI, AND Y. WAng, Large-time behavior of solutions to n-dimensional bipolar hydrodynamical models of semiconductors, SIAM J. Math. Anal., 43 (2011), pp. $1595-1630$.

[15] Y. Kuang, Delay Differential Equations with Applications in Population Dynamics, Academic 
Press, Boston, 1993.

[16] W.-T. Li, S. RuAn, AND Z.-C. WANG, On the diffusive Nicholson's blowflies equation with nonlocal delays, J. Nonlinear Sci., 17 (2007), pp. 505-525.

[17] C.-K. Lin AND M. MeI, On travelling wavefronts of the Nicholson's blowflies equations with diffusion, Proc. Roy. Soc. Edinburgh Sect. A, 140 (2010), pp. 135-152.

[18] G. Lv AND M.-X. WANG, Nonlinear stability of travelling wave fronts for delayed reaction diffusion equations, Nonlinearity, 23 (2010), pp. 1609-1630.

[19] S. MA, Traveling waves for non-local delayed diffusion equations via auxiliary equations, J. Differential Equations, 237 (2007), pp. 259-277.

[20] A. Matsumura And M. Mei, Convergence to travelling fronts of solutions of the p-system with viscosity in the presence of a boundary, Arch. Rational Mech. Anal., 146 (1999), pp. 1-22

[21] A. Matsumura And T. Nishida, The initial value problem for the equations of motion of viscous and heat-conductive gases, J. Math. Kyoto Univ., 20 (1980), pp. 67-104.

[22] M. MEI, Global smooth solutions of the Cauchy problem for higher-dimensional generalized pulse transmission equations, Acta Math. Appl. Sin., 14 (1991), pp. 450-461 (in Chinese).

[23] M. MeI, C.-K. Lin, C.-T. Lin, AND J. W.-H. So, Traveling wavefronts for time-delayed reaction-diffusion equation. I. Local nonlinearity, J. Differential Equations, 247 (2009), pp. $495-510$.

[24] M. MeI, C.-K. Lin, C.-T. Lin, And J. W.-H. So, Traveling wavefronts for time-delayed reaction-diffusion equation. II. Nonlocal nonlinearity, J. Differential Equations, 247 (2009), pp. $511-529$.

[25] M. Mei, C. Ou, And X.-Q. ZhaO, Global stability of monostable traveling waves for nonlocal time-delayed reaction-diffusion equations, SIAM J. Math. Anal., 42 (2010), pp. 2762-2790; erratum, SIAM J. Math. Anal., 44 (2012), pp. 538-540.

[26] M. Mei And B. Rubino, Convergence to traveling waves with decay rates for solutions of the initial boundary problem to a nonconvex relaxation model, J. Differential Equations, 159 (1999), pp. 138-185.

[27] M. Mei And J. W.-H. So, Stability of strong traveling waves for a nonlocal time-delayed reaction-diffusion equation, Proc. Roy. Soc. Edinburgh Sect. A, 138 (2008), pp. 551-568.

[28] M. Mei, J. W.-H. So, M.Y. Li, And S. S. P. Shen, Asymptotic stability of traveling waves for the Nicholson's blowflies equation with diffusion, Proc. Roy. Soc. Edinburgh Sect. A, 134 (2004), pp. 579-594.

[29] M. Mei AND Y. WANG, Remark on stability of traveling waves for nonlocal Fisher-KPP equations, Int. J. Num. Anal. Model. Ser. B, 2 (2011), pp. 379-401.

[30] A. J. Nicholson, Competition for food amongst Lucilia Cuprina larvae, in Proceedings of the 8th International Congress of Entomology, Stockholm, 1948, pp. 277-281.

[31] A. J. Nicholson, An outline of dynamics of animal populations, Aust. J. Zool., 2 (1954), pp. 9-65.

[32] K. W. SCHAAF, Asymptotic behavior and traveling wave solutions for parabolic functional differential equations, Trans. Amer. Math. Soc., 302 (1987), pp. 587-615.

[33] H. L. Sмiтh, An Introduction to Delay Differential Equations with Applications to the Life Sciences, Texts Appl. Math. 57, Springer, New York, 2011.

[34] J. So And Y. YAng, Dirichlet problem for the diffusive Nicholson's blowflies equation, J. Differential Equations, 150 (1998), pp. 317-348.

[35] J. So And X. Zou, Traveling waves for the diffusive Nicholson's blowflies equation, Appl. Math. Comput., 122 (2001), pp. 385-392.

[36] X. TAng And J. Shen, Oscillation of delay differential equations with variable coefficients, J. Math. Anal. Appl., 217 (1998), pp. 32-42.

[37] E. Trofimchuk, V. Tkachenko, and S. Trofimchuk, Slowly oscillating wave solutions of a single species reaction-diffusion equation with delay, J. Differential Equations, 245 (2008), pp. 2307-2332.

[38] E. Trofimchuk and S. Trofimchuk, Admissible wavefront speeds for a single species reactiondiffusion equation with delay, Discrete Contin. Dyn. Syst. Ser. A, 20 (2008), pp. 407-423.

[39] S.-L. WU, W.-T. LI, AND S.-Y. LiU, Asymptotic stability of traveling wave fronts in nonlocal reaction-diffusion equations with delay, J. Math. Anal. Appl., 360 (2009), pp. 439-458.

[40] S.-L. WU, H.-Q. ZhaO, AND S.-Y. LiU, Asymptotic stability of traveling waves for delayed reaction-diffusion equations with crossing-monostability, Z. Angew. Math. Phys. 62 (2011), pp. 377-397. 